C. Faber $\cdot$ R. Pandharipande

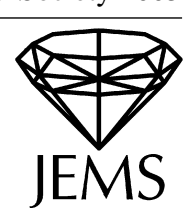

\title{
Relative maps and tautological classes
}

Received October 1, 2003 and in revised form April 12, 2004

\section{Introduction}

\subsection{Tautological classes}

Let $\bar{M}_{g, n}$ be the moduli space of stable curves of genus $g$ with $n$ marked points. Let $A^{*}\left(\bar{M}_{g, n}\right)$ denote the Chow ring with $\mathbb{Q}$-coefficients. The system of tautological rings is defined to be the set of smallest $\mathbb{Q}$-subalgebras of the Chow rings,

$$
R^{*}\left(\bar{M}_{g, n}\right) \subset A^{*}\left(\bar{M}_{g, n}\right),
$$

satisfying the following two properties:

(i) The system is closed under push-forward via all maps forgetting markings:

$$
\pi_{*}: R^{*}\left(\bar{M}_{g, n}\right) \rightarrow R^{*}\left(\bar{M}_{g, n-1}\right) .
$$

(ii) The system is closed under push-forward via all gluing maps:

$$
\begin{gathered}
\iota_{*}: R^{*}\left(\bar{M}_{g_{1}, n_{1} \cup\{*\}}\right) \otimes_{\mathbb{Q}} R^{*}\left(\bar{M}_{g_{2}, n_{2} \cup\{\bullet\}}\right) \rightarrow R^{*}\left(\bar{M}_{g_{1}+g_{2}, n_{1}+n_{2}}\right), \\
\iota_{*}: R^{*}\left(\bar{M}_{g, n \cup\{*, \bullet\}}\right) \rightarrow R^{*}\left(\bar{M}_{g+1, n}\right),
\end{gathered}
$$

with attachments along the markings $*$ and $\bullet$.

While the definition appears restrictive, natural algebraic constructions typically yield Chow classes lying in the tautological ring. For example, the standard $\psi, \kappa$, and $\lambda$ classes in $A^{*}\left(\bar{M}_{g, n}\right)$ all lie in the tautological ring. The tautological rings also possess a rich conjectural structure; see [FP] for a detailed discussion.

The cotangent line classes $\psi$ are tautological by the following construction. For each marking $i$, let $L_{i}$ denote the associated cotangent line bundle over $\bar{M}_{g, n}$. The class $\psi_{i}$ is the first Chern class of $L_{i}$,

$$
\psi_{i}=c_{1}\left(L_{i}\right) \in A^{1}\left(\bar{M}_{g, n}\right) .
$$

Let $\pi$ denote the map forgetting the last marking,

$$
\pi: \bar{M}_{g, n+1} \rightarrow \bar{M}_{g, n},
$$

C. Faber: Institutionen för Matematik, Kungliga Tekniska Högskolan, 10044 Stockholm, Sweden; e-mail: faber@math.kth.se

R. Pandharipande: Department of Mathematics, Princeton University, Princeton, NJ 08544, USA; e-mail: rahulp@math.princeton.edu 
and let $\iota$ denote the gluing map,

$$
\iota: \bar{M}_{g,\{1,2, \ldots, i-1, *, i+1, \ldots, n\}} \times \bar{M}_{0,\{\bullet, i, n+1\}} \rightarrow \bar{M}_{g, n+1} .
$$

The $\mathbb{Q}$-multiples of the fundamental classes $\left[\bar{M}_{g, n}\right]$ are contained in the tautological rings (as $\mathbb{Q}$-multiples of the units in the subalgebras). A direct calculation shows that

$$
-\pi_{*}\left(\left(\iota_{*}\left(\left[\bar{M}_{g, n}\right] \times\left[\bar{M}_{0,3}\right]\right)\right)^{2}\right)=\psi_{i} .
$$

Hence, the cotangent line classes lie in the tautological rings. A discussion of the $\kappa$ and $\lambda$ classes can be found in [FP].

Gromov-Witten theory defines natural classes in $A^{*}\left(\bar{M}_{g, n}\right)$. Let $X$ be a nonsingular projective variety, and let $\bar{M}_{g, n}(X, \beta)$ be the moduli space of stable maps representing $\beta \in H_{2}(X, \mathbb{Z})$. Let $\rho$ denote the map to the moduli of curves,

$$
\rho: \bar{M}_{g, n}(X, \beta) \rightarrow \bar{M}_{g, n},
$$

in case $2 g-2+n>0$. Let $\omega \in A^{*}\left(\bar{M}_{g, n}(X, \beta)\right)$ be a Gromov-Witten class composed of algebraic primary fields and descendents. Then

$$
\rho_{*}\left(\omega \cap\left[\bar{M}_{g, n}(X, \beta)\right]^{\mathrm{vir}}\right) \in A^{*}\left(\bar{M}_{g, n}\right) .
$$

The push-forwards of all Gromov-Witten classes of compact homogeneous varieties $X$ lie in the tautological ring by the localization formula for the virtual class (see [GrP1]). We do not know any example defined over $\overline{\mathbb{Q}}$ of a Gromov-Witten class for which the push-forward is not tautological.

The moduli spaces of Hurwitz covers of $\mathbf{P}^{1}$ also define natural classes on the moduli space of curves. Let $g \geq 0$. Let $\mu^{1}, \ldots, \mu^{m}$ be $m$ partitions of equal size $d$ satisfying

$$
2 g-2+2 d=\sum_{i=1}^{m}\left(d-\ell\left(\mu^{i}\right)\right),
$$

where $\ell\left(\mu^{i}\right)$ denotes the length of the partition $\mu^{i}$. The moduli space of Hurwitz covers,

$$
H_{g}\left(\mu^{1}, \ldots, \mu^{m}\right),
$$

parameterizes morphisms

$$
f: C \rightarrow \mathbf{P}^{1},
$$

where $C$ is a complete, connected, nonsingular curve with marked profiles $\mu^{1}, \ldots, \mu^{m}$ over $m$ ordered points of the target (and no ramifications elsewhere). Two Hurwitz covers

$$
C \stackrel{f}{\rightarrow} \mathbf{P}^{1}, \quad C^{\prime} \stackrel{f^{\prime}}{\rightarrow} \mathbf{P}^{1}
$$

are isomorphic if there exist isomorphisms

$$
\alpha: C \rightarrow C^{\prime}, \quad \beta: \mathbf{P}^{1} \rightarrow \mathbf{P}^{1}
$$

which commute with $f, f^{\prime}$ and respect all the markings. The moduli space of Hurwitz covers is a dense open set of the compact moduli space of admissible covers [HM],

$$
H_{g}\left(\mu^{1}, \ldots, \mu^{m}\right) \subset \bar{H}_{g}\left(\mu^{1}, \ldots, \mu^{m}\right) .
$$


Let $\rho$ denote the map to the moduli of curves,

$$
\rho: \bar{H}_{g}\left(\mu^{1}, \ldots, \mu^{m}\right) \rightarrow \bar{M}_{g, \sum_{i=1}^{m} \ell\left(\mu^{i}\right)} .
$$

The push-forwards of the fundamental classes,

$$
\rho_{*}\left(\bar{H}_{g}\left(\mu^{1}, \ldots, \mu^{m}\right)\right) \in A^{*}\left(\bar{M}_{g, \sum_{i=1}^{m} \ell\left(\mu^{i}\right)}\right),
$$

define classes on the moduli of curves.

The following two questions provide classical motivation for the results of our paper:

(i) Are the push-forwards $\rho_{*}\left(\bar{H}_{g}\left(\mu^{1}, \ldots, \mu^{m}\right)\right)$ tautological?

(ii) Can the push-forwards be computed?

We settle (i)-(ii) in the affirmative.

While Hurwitz covers have played a basic role in the study of the moduli of curves, questions (i)-(ii) were open even for the class of the hyperelliptic locus,

$$
\rho_{*}\left(\bar{H}_{g}((2), \ldots,(2))\right) \text {, }
$$

in $\bar{M}_{g, 2 g+2}$.

\subsection{Stable relative maps}

0.2.1. Overview. We study Hurwitz covers in the much richer context of stable relative maps to $\mathbf{P}^{1}$. Stable relative maps combine features of stable maps and admissible covers. The moduli space of stable relative maps was first introduced by $\mathrm{Li}$ and Ruan in [LR]. An algebraic development can be found in [Li]. The main results of the paper concern the relationship between tautological classes on the moduli space of stable relative maps and the moduli space of curves.

0.2.2. The parameterized case. We first define stable relative maps to a parameterized $\mathbf{P}^{1}$. Let $g, n \geq 0$. Let $\mu^{1}, \ldots, \mu^{m}$ be $m$ partitions of equal size $d>0$. A stable relative map

$$
\left[\left(C, p_{1}, \ldots, p_{n}, Q_{1}, \ldots, Q_{m}\right) \stackrel{f}{\rightarrow}\left(T, q_{1}, \ldots, q_{m}\right) \stackrel{\epsilon}{\rightarrow} \mathbf{P}^{1}\right] \in \bar{M}_{g, n}^{\dagger}\left(\mu^{1}, \ldots, \mu^{m}\right)
$$

consists of the following data:

(i) $T$ is a complete, connected genus 0 nodal curve with $m$ distinct nonsingular markings $q_{1}, \ldots, q_{m}$.

(ii) The structure map $\epsilon: T \rightarrow \mathbf{P}^{1}$ restricts to an isomorphism on a unique component $P \subset T$ and contracts all other components of $T$.

(iii) All extremal components of the tree $T$ not equal to $P$ carry at least one of the $m$ markings.

(iv) $C$ is a complete, connected genus $g$ nodal curve with $n+\sum_{i=1}^{m} \ell\left(\mu^{i}\right)$ distinct nonsingular markings

$$
\left\{p_{1}, \ldots, p_{n}\right\} \cup \bigcup_{i=1}^{m} Q_{i}
$$

where $\left|Q_{i}\right|=\ell\left(\mu^{i}\right)$. 
(v) The morphism $f$ satisfies the following basic conditions:

(a) $f$ satisfies admissible cover conditions over the nodes of $T$ : matching branchings with no markings or contracted components of $C$ lying over the nodes of $T$,

(b) $f$ has profile $\mu^{i}$ over $q_{i}$,

(c) $Q_{i}$ is a complete marking of the fiber of $f$ over $q_{i}$ with $\ell\left(\mu^{i}\right)$ distinct points.

(vi) The data has a finite automorphism group. The automorphism group is determined by curve automorphisms $\alpha: C \rightarrow C$ and $\beta: T \rightarrow T$ which respect the markings and commute with $f, \epsilon$, and the identity map on $\mathbf{P}^{1}$.

The data $C \stackrel{f}{\rightarrow} T \stackrel{\epsilon}{\rightarrow} \mathbf{P}^{1}$ and $C^{\prime} \stackrel{f^{\prime}}{\rightarrow} T^{\prime} \stackrel{\epsilon^{\prime}}{\rightarrow} \mathbf{P}^{1}$ are isomorphic if there exist isomorphisms $\alpha: C \rightarrow C^{\prime}$ and $\beta: T \rightarrow T^{\prime}$, which respect all the markings and commute with $f, f^{\prime}, \epsilon, \epsilon^{\prime}$, and the identity map on $\mathbf{P}^{1}$. Condition (vi) may be interpreted as a stability condition.

The superscript $\uparrow$ in the notation for the moduli space of stable relative maps,

$$
\bar{M}_{g, n}^{\dagger}\left(\mu^{1}, \ldots, \mu^{m}\right)
$$

indicates the target $\mathbf{P}^{1}$ is parameterized. The moduli space, a Deligne-Mumford stack, admits several canonical structures:

(i) a virtual fundamental class

$$
\left[\bar{M}_{g, n}^{\dagger}\left(\mu^{1}, \ldots, \mu^{m}\right)\right]^{\mathrm{vir}} \in A_{e}\left(\bar{M}_{g, n}^{\dagger}\left(\mu^{1}, \ldots, \mu^{m}\right)\right)
$$

in the expected dimension

$$
e=2 g-2+2 d+n+\sum_{i=1}^{m}\left(1+\ell\left(\mu^{i}\right)-d\right),
$$

(ii) evaluation maps

$$
\mathrm{ev}: \bar{M}_{g, n}^{\dagger}\left(\mu^{1}, \ldots, \mu^{m}\right) \rightarrow \mathbf{P}^{1}
$$

determined by the markings $p_{1}, \ldots, p_{n}$ and $q_{1}, \ldots, q_{m}$,

(iii) cotangent line classes $\psi \in A^{1}\left(\bar{M}_{g, n}^{\dagger}\left(\mu^{1}, \ldots, \mu^{m}\right)\right)$ determined by the markings $p_{1}, \ldots, p_{n}$ and $q_{1}, \ldots, q_{m}$,

(iv) a map to the moduli of curves (via the domain),

$$
\rho: \bar{M}_{g, n}^{\dagger}\left(\mu^{1}, \ldots, \mu^{m}\right) \rightarrow \bar{M}_{g, n+\sum_{i=1}^{m} \ell\left(\mu^{i}\right)},
$$

in case $2 g-2+n+\sum_{i=1}^{m} \ell\left(\mu^{i}\right)>0$,

(v) a map to the Fulton-MacPherson parameter space of points on $\mathbf{P}^{1}$ (via the target),

$$
\rho_{0}: \bar{M}_{g, n}^{\dagger}\left(\mu^{1}, \ldots, \mu^{m}\right) \rightarrow \mathbf{P}^{1}[m] .
$$

A relative Gromov-Witten class on the moduli space of stable relative maps,

$$
\omega=\prod_{i=1}^{n} \mathrm{ev}_{p_{i}}^{*}\left(\gamma_{i}\right) \psi_{p_{i}}^{k_{i}} \cup \prod_{j=1}^{m} \mathrm{ev}_{q_{j}}^{*}\left(\gamma_{j}^{\prime}\right) \psi_{q_{j}}^{k_{j}^{\prime}},
$$

is constructed from structures (ii)-(iii). Here, $\gamma_{i}, \gamma_{j}^{\prime} \in A^{*}\left(\mathbf{P}^{1}\right)$. 
The map to the moduli of curves (iv) may be defined to exist in all cases relevant to our study. We will only consider moduli spaces of stable relative maps for which $m>0$. Since $d$ is positive, $\ell\left(\mu^{i}\right)>0$. Therefore, the inequality

$$
2 g-2+n+\sum_{i=1}^{m} \ell\left(\mu^{i}\right)>0
$$

can be violated only if $g=0$. The map to the moduli of curves is defined in the unstable genus 0 cases by viewing $\bar{M}_{0,1}$ and $\bar{M}_{0,2}$ as points.

0.2.3. The unparameterized case. We will also require stable relative maps to an unparameterized $\mathbf{P}^{1}$. Let $g, n \geq 0$. Let $\mu^{1}, \ldots, \mu^{m}$ be $m \geq 2$ partitions of equal size $d>0$. A stable relative map

$$
\left[\left(C, p_{1}, \ldots, p_{n}, Q_{1}, \ldots, Q_{m}\right) \stackrel{f}{\rightarrow}\left(T, q_{1}, \ldots, q_{m}\right)\right] \in \bar{M}_{g, n}\left(\mu^{1}, \ldots, \mu^{m}\right)
$$

consists of the following data:

(i) $T$ is a complete, connected genus 0 nodal curve with $m$ distinct nonsingular markings $q_{1}, \ldots, q_{m}$.

(ii) All extremal components of the tree $T$ carry at least one of the $m$ markings.

(iii) $C$ is a complete, connected genus $g$ nodal curve with $n+\sum_{i=1}^{m} \ell\left(\mu^{i}\right)$ distinct nonsingular markings $\left\{p_{1}, \ldots, p_{n}\right\} \cup \bigcup_{i=1}^{m} Q_{i}$, where $\left|Q_{i}\right|=\ell\left(\mu^{i}\right)$.

(iv) The morphism $f$ satisfies (a)-(c) of the parameterized definition.

(v) The data has a finite automorphism group. The automorphism group is determined by curve automorphisms $\alpha: C \rightarrow C$ and $\beta: T \rightarrow T$ which respect the markings and commute with $f$.

We will consider the unparameterized case only when $m \geq 2$. The moduli space $\bar{M}_{g, n}\left(\mu^{1}, \ldots, \mu^{m}\right)$ admits the following structures:

(i) a virtual fundamental class

$$
\left[\bar{M}_{g, n}\left(\mu^{1}, \ldots, \mu^{m}\right)\right]^{\mathrm{vir}} \in A_{e}\left(\bar{M}_{g, n}\left(\mu^{1}, \ldots, \mu^{m}\right)\right)
$$

in the expected dimension

$$
e=2 g-5+2 d+n+\sum_{i=1}^{m}\left(1+\ell\left(\mu^{i}\right)-d\right),
$$

(ii) cotangent line classes $\psi \in A^{1}\left(\bar{M}_{g, n}\left(\mu^{1}, \ldots, \mu^{m}\right)\right)$ determined by the markings $p_{1}, \ldots, p_{n}$ and $q_{1}, \ldots, q_{m}$,

(iii) a map to the moduli of curves (via the domain),

$$
\rho: \bar{M}_{g, n}\left(\mu^{1}, \ldots, \mu^{m}\right) \rightarrow \bar{M}_{g, n+\sum_{i=1}^{m} \ell\left(\mu^{i}\right)},
$$

in case $2 g-2+n+\sum_{i=1}^{m} \ell\left(\mu^{i}\right)>0$,

(iv) a map to the moduli of genus 0 pointed curves (via the target),

$$
\rho_{0}: \bar{M}_{g, n}\left(\mu^{1}, \ldots, \mu^{m}\right) \rightarrow \bar{M}_{0, m},
$$

in case $m \geq 3$. 
The moduli spaces of admissible covers arise as special cases of the moduli spaces of stable relative maps:

$$
\bar{M}_{g, 0}\left(\mu^{1}, \ldots, \mu^{m}\right)=\bar{H}_{g}\left(\mu^{1}, \ldots, \mu^{m}\right),
$$

in case

$$
2 g-2+2 d=\sum_{i=1}^{m}\left(d-\ell\left(\mu^{i}\right)\right) .
$$

Here, the virtual class of the space of stable relative maps (i) is equal to the fundamental class of the space of admissible covers.

A relative Gromov-Witten class on the moduli space of stable relative maps,

$$
\omega=\prod_{i=1}^{n} \psi_{p_{i}}^{k_{i}} \cup \prod_{j=1}^{m} \psi_{q_{j}}^{k_{j}^{\prime}},
$$

is constructed from the cotangent line classes (ii). The evaluation maps are lost in the unparameterized case. The map to the moduli of curves (iii) is defined in all relevant cases as before.

0.2.4. Results. We prove results which show the compatibility of Gromov-Witten classes on the moduli of stable relative maps and tautological classes on the moduli of curves in both the parameterized and unparameterized cases.

Theorem 1. Relative Gromov-Witten classes push forward to tautological classes:

(i) For every relative Gromov-Witten class $\omega$ on $\bar{M}_{g, n}^{\dagger}\left(\mu^{1}, \ldots, \mu^{m}\right)$,

$$
\rho_{*}\left(\omega \cap\left[\bar{M}_{g, n}^{\dagger}\left(\mu^{1}, \ldots, \mu^{m}\right)\right]^{\text {vir }}\right)
$$

is a tautological class on the target moduli space of curves.

(ii) For every relative Gromov-Witten class $\omega$ on $\bar{M}_{g, n}\left(\mu^{1}, \ldots, \mu^{m}\right)$,

$$
\rho_{*}\left(\omega \cap\left[\bar{M}_{g, n}\left(\mu^{1}, \ldots, \mu^{m}\right)\right]^{\mathrm{vir}}\right)
$$

is a tautological class on the target moduli space of curves.

Theorem 1 is proven by studying relations obtained by virtual localization. The proof for both parts is constructive: the push-forwards of relative Gromov-Witten classes are recursively calculated in the tautological ring in terms of the standard $\psi, \kappa$, and $\lambda$ classes. The proof is given in Sections 2 and 3 of the paper. In practice, the execution of the recursion is computationally quite hard. A discussion (together with a calculation) can be found in Section 2.5 .6

\subsection{Consequences}

Theorem 1 has several consequences for the geometry of the moduli space of curves.

Proposition 1. The moduli space of Hurwitz covers yields tautological classes,

$$
\rho_{*}\left(\bar{H}_{g}\left(\mu^{1}, \ldots, \mu^{m}\right)\right) \in R^{*}\left(\bar{M}_{g, \sum_{i=1}^{m} \ell\left(\mu^{i}\right)}\right) .
$$


Proposition 1 follows from part (ii) of Theorem 1 in case $\omega$ is the identity class and the space of stable relative maps is specialized to the space of admissible covers. Moreover, the push-forwards are effectively determined.

An additive set of generators for $R^{*}\left(\bar{M}_{g, n}\right)$ is indexed by strata dual graphs decorated with $\psi$ and $\kappa$ classes on the nodes (see [GrP2]). We review the basic push-pull method for constructing relations in $R^{*}\left(\bar{M}_{g, n}\right)$ introduced in [P1], [BP]. Consider the two maps to the moduli of curves defined for the moduli space of admissible covers:

$$
\bar{M}_{0, m} \stackrel{\rho_{0}}{\longleftarrow} \bar{H}_{g}\left(\mu^{1}, \ldots, \mu^{m}\right) \stackrel{\rho}{\longrightarrow} \bar{M}_{g, \sum_{i=1}^{n} \ell} \ell\left(\mu^{i}\right) \text {. }
$$

Let $r$ denote a relation among boundary strata in $\bar{M}_{0, m}$. Then

$$
\rho_{*} \rho_{0}^{*}(r)
$$

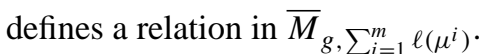

Relation (1), however, consists of push-forwards of fundamental classes of auxiliary moduli spaces of admissible covers. The push-pull method, along with a case-by-case analysis of the push-forwards, yields the basic relations in genus 1 and 2 among descendent stratum classes (see [P1], [BP], [Ge1], [Ge2]). Proposition 1] now guarantees all push-forwards arising in $\sqrt{1})$ lie in $R^{*}\left(\bar{M}_{g, n}\right)$. The push-pull method together with Proposition 1 provides a rich source of tautological relations in the moduli space of curves.

Speculation 1. All relations in the tautological ring are obtained via the push-pull method and Proposition 1 .

A further study of the push-pull relations was undertaken in [I] $]$. In the absence of Proposition 1 , only the principal terms (the symbols) of the relations could be studied. The main result of $[\mathrm{I}]$ is of interest here:

(i) Ionel's vanishing,

$$
\prod_{i=1}^{n} \psi_{i}^{e_{i}} \prod_{j \geq 0} \kappa_{j}^{f_{j}}=0 \in R^{*}\left(M_{g, n}\right) \quad \text { for } \sum_{i=1}^{n} e_{i}+\sum_{j \geq 0} j f_{j} \geq g+\delta_{0 g}-\delta_{0 n},
$$

can be obtained from the symbols of push-pull relations.

The above vanishing generalizes two well-known vanishings:

(ii) Getzler's (conjectured) vanishing,

$$
\prod_{i=1}^{n} \psi_{i}^{e_{i}}=0 \in R^{*}\left(M_{g, n}\right) \quad \text { for } \sum_{i=1}^{n} e_{i} \geq g+\delta_{0 g},
$$

(iii) Looijenga's vanishing,

$$
\prod_{j \geq 0} \kappa_{j}^{f_{j}}=0 \in R^{*}\left(M_{g}\right) \quad \text { for } \sum_{j \geq 0} j f_{j} \geq g-1 .
$$

The tautological ring, $R^{*}\left(M_{g}\right) \subset R^{*}\left(\bar{M}_{g}\right)$, for the open moduli space $M_{g}$, is defined to be the image of $R^{*}\left(\bar{M}_{g}\right)$ via the natural map

$$
R^{*}\left(\bar{M}_{g}\right) \subset A^{*}\left(\bar{M}_{g}\right) \rightarrow A^{*}\left(M_{g}\right) .
$$


Let $\partial \bar{M}_{g}=\bar{M}_{g} \backslash M_{g}$ denote the boundary of the moduli space of curves. The tautological classes of the boundary, $R^{*}\left(\partial \bar{M}_{g}\right) \subset A^{*}\left(\partial \bar{M}_{g}\right)$, are defined by the push-forwards of all tautological classes on the normalized boundary divisors via the gluing morphisms. There is a restriction sequence

$$
R^{*}\left(\partial \bar{M}_{g}\right) \rightarrow R^{*}\left(\bar{M}_{g}\right) \rightarrow R^{*}\left(M_{g}\right) \rightarrow 0,
$$

for which the exactness in the middle is unknown. An identical discussion holds for $\bar{M}_{g, n}$.

Proposition 1 combined with the symbol analysis of [I] directly yields a much stronger version of Ionel's vanishings.

Proposition 2. The class $\prod_{i=1}^{n} \psi_{i}^{e_{i}} \prod_{j \geq 0} \kappa_{j}^{f_{j}}$ lies in the image

$$
\operatorname{Im} R^{*}\left(\partial \bar{M}_{g, n}\right) \subset R^{*}\left(\bar{M}_{g, n}\right)
$$

for $\sum_{i=1}^{n} e_{i}+\sum_{j \geq 0} j f_{j} \geq g+\delta_{0 g}-\delta_{0 n}$.

Proposition 2 has consequences beyond Ionel's original vanishing. A uniform Gorenstein conjecture was advanced in $[\overline{\mathrm{FP}}],[\overline{\mathrm{P}} 2]$ for the tautological rings of $\bar{M}_{g, n}$ and the quotients corresponding to the moduli spaces of compact type curves $M_{g, n}^{\mathrm{c}}$ and curves with rational tails $M_{g, n}^{\mathrm{rt}}$. An inductive argument using Proposition 2 yields the following result.

Proposition 3. The socle and vanishing claims of the Gorenstein conjectures hold for $\bar{M}_{g, n}, M_{g, n}^{\mathrm{c}}$, and $M_{g, n}^{\mathrm{rt}}$.

Proposition 3 is proven in Section 4.1. A different approach to Proposition 3 has been pursued recently by Graber and Vakil [GrV1], [GrV2].

Another consequence of Proposition 2 is the exactness of the restriction sequence

$$
R^{*}\left(\partial \bar{M}_{g}\right) \rightarrow R^{*}\left(\bar{M}_{g}\right) \rightarrow R^{*}\left(M_{g}\right) \rightarrow 0
$$

in degrees greater than $g-2$. The result motivates the following conjecture.

Conjecture 2. The restriction sequence (2) is exact in all degrees.

More generally, we conjecture the exactness of the tautological sequences associated to the compact type and rational tail moduli spaces,

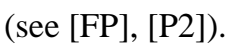

$$
\begin{aligned}
& R^{*}\left(\bar{M}_{g, n} \backslash M_{g, n}^{\mathrm{c}}\right) \rightarrow R^{*}\left(\bar{M}_{g, n}\right) \rightarrow R^{*}\left(M_{g, n}^{\mathrm{c}}\right) \rightarrow 0, \\
& R^{*}\left(\bar{M}_{g, n} \backslash M_{g, n}^{\mathrm{rt}}\right) \rightarrow R^{*}\left(\bar{M}_{g, n}\right) \rightarrow R^{*}\left(M_{g, n}^{\mathrm{rt}}\right) \rightarrow 0,
\end{aligned}
$$

Finally, Proposition 2 is the required form of Getzler's vanishing for applications to Gromov-Witten theory. The main consequence is the following reconstruction result proved in Section 4.2 .

Proposition 4. Let $X$ be a nonsingular projective variety. All descendent Gromov-Witten invariants of $X$ can be reconstructed from the restricted invariants

$$
\left\langle\tau_{e_{1}}\left(\gamma_{1}\right) \cdots \tau_{e_{n}}\left(\gamma_{n}\right) \prod_{j \geq 0} \kappa_{j}^{f_{j}}\right\rangle_{g, n, \beta}^{X},
$$

where $\sum_{i=1}^{n} e_{i}+\sum_{j \geq 0} j f_{j}<g+\delta_{0 g}$. 
We conjecture a stronger result based on a new conjectured generation statement for $R^{*}\left(M_{g, n}\right)$ and the conjectured exactness of tautological restriction sequences. The conjectural framework is discussed in Section 4.3 .

Conjecture 3. All descendent Gromov-Witten invariants of $X$ can be reconstructed from the restricted invariants

where $\sum_{i=1}^{n} e_{i}<g+\delta_{0 g}$.

$$
\left\langle\tau_{e_{1}}\left(\gamma_{1}\right) \cdots \tau_{e_{n}}\left(\gamma_{n}\right)\right\rangle_{g, n, \beta}^{X},
$$

\section{Localization}

\subsection{Overview}

The localization formula for the virtual class of the moduli space $\bar{M}_{g, n}^{\dagger}\left(\mu^{1}, \ldots, \mu^{m}\right)$ is required for our proof of Theorem 1 . The localization formula can be obtained from the algebraic construction of the virtual class [Li] together with the virtual localization formula [GrP1]. A derivation can be found in [GrV2].

\subsection{Disconnected domains}

1.2.1. Notation. We will require moduli spaces of stable relative maps with disconnected domains in both the parameterized and unparameterized cases. The definitions follow the connected case with minor variations.

We first introduce notation for the disconnected case. Let $\mathbf{g}$ be an ordered set of genera,

$$
\mathbf{g}=\left(g_{1}, \ldots, g_{c}\right) .
$$

Let $\mathbf{n}$ be an ordered set partition,

$$
\mathbf{n}=\left(n_{1}, \ldots, n_{c}\right), \quad \bigcup_{i=1}^{c} n_{i}=\underline{n},
$$

where $\underline{n}=\{1, \ldots, n\}$. The data $\mathbf{g}$ and $\mathbf{n}$ describe the genera and marking distributions of a disconnected domain with $c$ ordered components.

The degree distribution on a disconnected domain is described by an ordered partition $\mathbf{d}$,

$$
\mathbf{d}=\left(d_{1}, \ldots, d_{c}\right), \quad d_{i}>0, \quad \sum_{i} d_{i}=d .
$$

A partition $\mu$ of $d$ of type $\mathbf{d}$ is an ordered set of partitions,

$$
\mu=(\mu[1], \ldots, \mu[c]),
$$

where $\mu[i]$ is a partition of $d_{i}$. Ordered partitions of type $\mathbf{d}$ describe ramification conditions on the disconnected domain.

1.2.2. Moduli spaces. We first define stable relative maps to a parameterized $\mathbf{P}^{1}$ with disconnected domains. Let $\mathbf{g}$, $\mathbf{n}$, and $\mathbf{d}$ be as defined above. Let $\mu^{1}, \ldots, \mu^{m}$ be $m$ parti- 
tions of $d$ of type $\mathbf{d}$. A stable relative map

$$
\left[\left(C, p_{1}, \ldots, p_{n}, Q_{1}, \ldots, Q_{m}\right) \stackrel{f}{\rightarrow}\left(T, q_{1}, \ldots, q_{m}\right) \stackrel{\epsilon}{\rightarrow} \mathbf{P}^{1}\right] \in \bar{M}_{\mathbf{g}, \mathbf{n}}^{\dagger}\left(\mu^{1}, \ldots, \mu^{m}\right)
$$

consists of the data (i)-(vi) of Section 0.2 .2 with (iv) replaced by:

(iv) $C$ is a complete, disconnected nodal curve with $c$ ordered components carrying $n+\sum_{i=1}^{m} \ell\left(\mu^{i}\right)$ distinct nonsingular markings $\left\{p_{1}, \ldots, p_{n}\right\} \cup \bigcup_{i=1}^{m} Q_{i}$, where $\left|Q_{i}\right|=\ell\left(\mu^{i}\right)$. The genera of the components are determined by $\mathbf{g}$. The markings are distributed by the data of $\mathbf{n}$ and $\mu^{i}$.

The data $C \stackrel{f}{\rightarrow} T \stackrel{\epsilon}{\rightarrow} \mathbf{P}^{1}$ and $C^{\prime} \stackrel{f^{\prime}}{\rightarrow} T^{\prime} \stackrel{\epsilon^{\prime}}{\rightarrow} \mathbf{P}^{1}$ are isomorphic if there exist isomorphisms $\alpha: C \rightarrow C^{\prime}$ and $\beta: T \rightarrow T^{\prime}$ which respect all the structures and commute with $f, f^{\prime}, \epsilon, \epsilon^{\prime}$, and the identity map on $\mathbf{P}^{1}$.

The moduli space, a Deligne-Mumford stack, admits the canonical structures (i)(iv) of the connected case. Relative Gromov-Witten classes are defined as before using structures (ii)-(iii). The map to the moduli of curves (iv) via the domain is slightly altered:

$$
\rho: \bar{M}_{\mathbf{g}, \mathbf{n}}^{\dagger}\left(\mu_{1}, \ldots, \mu_{m}\right) \rightarrow \prod_{i=1}^{c} \bar{M}_{g_{i},\left|n_{i}\right|+\sum_{j=1}^{m} \ell\left(\mu^{j}[i]\right)}
$$

in case

$$
2 g_{i}-2+\left|n_{i}\right|+\sum_{j=1}^{m} \ell\left(\mu^{j}[i]\right)>0
$$

for all $i$. Since $m>0$ and $d_{i}>0$, the map to the moduli of curves is defined in all relevant cases by viewing $\bar{M}_{0,1}$ and $\bar{M}_{0,2}$ as points.

The definition of the unparameterized moduli space $\bar{M}_{\mathbf{g}, \mathbf{n}}\left(\mu^{1}, \ldots, \mu^{m}\right)$ is obtained similarly. Part (iii) of the definition of Section 0.2 .3 is modified to allow disconnected domains. Also, the canonical map to the moduli of curves (iii) is replaced by a map to a product of moduli spaces.

Of course, when $c=1$, the moduli spaces of stable relative maps with connected domains are recovered.

1.2.3. Theorem 1 revisited. We will prove a stronger form of Theorem 1 including all the moduli spaces of stable relative maps with disconnected domains.

Theorem 2. Relative Gromov-Witten classes push-forward to tautological classes:

(i) For every relative Gromov-Witten class $\omega$ on $\bar{M}_{\mathbf{g}, \mathbf{n}}^{\dagger}\left(\mu^{1}, \ldots, \mu^{m}\right)$,

$$
\rho_{*}\left(\omega \cap\left[\bar{M}_{\mathbf{g}, \mathbf{n}}^{\dagger}\left(\mu^{1}, \ldots, \mu^{m}\right)\right]^{\mathrm{vir}}\right)
$$

is a tautological class on the target.

(ii) For every relative Gromov-Witten class $\omega$ on $\bar{M}_{\mathbf{g}, \mathbf{n}}\left(\mu^{1}, \ldots, \mu^{m}\right)$,

$$
\rho_{*}\left(\omega \cap\left[\bar{M}_{\mathbf{g}, \mathbf{n}}\left(\mu^{1}, \ldots, \mu^{m}\right)\right]^{\mathrm{vir}}\right)
$$

is a tautological class on the target. 


\subsection{Torus actions, fixed points, and the localization formula}

1.3.1. The torus action. The equivariant Chow ring of $\mathbb{C}^{*}$ is freely generated by the first Chern class $t$ of the standard representation,

$$
A_{\mathbb{C}^{*}}^{*}([\text { point }])=\mathbb{Q}[t] .
$$

Let $\mathbb{C}^{*}$ act diagonally on a 2-dimensional vector space $V$ via the trivial and standard representations,

$$
\xi \cdot\left(v_{1}, v_{2}\right)=\left(v_{1}, \xi \cdot v_{2}\right)
$$

Let $\mathbf{P}^{1}=\mathbf{P}(V)$. Let $0, \infty$ be the fixed points $[1,0],[0,1]$ of the corresponding $\mathbb{C}^{*}$-action on $\mathbf{P}(V)$.

An equivariant lifting of $\mathbb{C}^{*}$ to a line bundle $L$ over $\mathbf{P}(V)$ is uniquely determined by the fiber representations at the fixed points $L_{0}$ and $L_{\infty}$. The canonical lifting of $\mathbb{C}^{*}$ to the tangent bundle $T_{\mathbf{P}}$ has weights $[t,-t]$.

The representation (3) canonically induces a $\mathbb{C}^{*}$-action on the moduli space $\bar{M}_{\mathbf{g}, \mathbf{n}}\left(\mu^{1}, \ldots, \mu^{m}\right)$ by translation of the map:

$$
\xi \cdot\left[C \stackrel{f}{\rightarrow} T \stackrel{\epsilon}{\rightarrow} \mathbf{P}^{1}\right]=\left[C \stackrel{f}{\rightarrow} T \stackrel{\xi \cdot \epsilon}{\rightarrow} \mathbf{P}^{1}\right] .
$$

The canonical structures of the moduli space of stable relative maps are compatible with the induced $\mathbb{C}^{*}$-action. The virtual fundamental class canonically lifts to equivariant Chow theory,

$$
\left[\bar{M}_{\mathbf{g}, \mathbf{n}}^{\dagger}\left(\mu^{1}, \ldots, \mu^{m}\right)\right]^{\mathrm{vir}} \in A_{e}^{\mathbb{C}^{*}}\left(\bar{M}_{\mathbf{g}, \mathbf{n}}^{\dagger}\left(\mu^{1}, \ldots, \mu^{m}\right)\right) .
$$

The $\mathbb{C}^{*}$-action on the moduli space canonically lifts to the cotangent line bundles and is equivariant with respect to the evaluation maps. Therefore, equivariant relative GromovWitten classes

$$
\omega=\prod_{i=1}^{n} \mathrm{ev}_{p_{i}}^{*}\left(\gamma_{i}\right) \psi_{p_{i}}^{k_{i}} \cup \prod_{j=1}^{m} \mathrm{ev}_{q_{j}}^{*}\left(\gamma_{j}^{\prime}\right) \psi_{q_{j}}^{k_{j}^{\prime}}
$$

are well defined for $\gamma_{i}, \gamma_{j}^{\prime} \in A_{\mathbb{C}^{*}}^{*}\left(\mathbf{P}^{1}\right)$. The $\mathbb{C}^{*}$-action on the moduli space is equivariant (via $\rho$ ) with respect to the trivial action on the moduli space of curves.

We will require a localization formula for the equivariant push-forward:

$$
\rho_{*}\left(\omega \cap\left[\bar{M}_{\mathbf{g}, \mathbf{n}}^{\dagger}\left(\mu^{1}, \ldots, \mu^{m}\right)\right]^{\mathrm{vir}}\right) .
$$

1.3.2. Graph notation. Let the data

$$
\mathbf{g}=\left(g_{1}, \ldots, g_{c}\right), \quad \mathbf{n}=\left(n_{1}, \ldots, n_{c}\right), \quad \mathbf{d}=\left(d_{1}, \ldots, d_{c}\right)
$$

describe (possibly) disconnected domains with $c$ components. The torus fixed loci of $\bar{M}_{\mathbf{g}, \mathbf{n}}^{\dagger}\left(\mu^{1}, \ldots, \mu^{m}\right)$ are indexed by localization graphs,

$$
\Gamma=\left(V, E, N, \gamma, \pi, \delta,\left(R^{1}, \ldots, R^{m}\right)\right),
$$

defined by the following conditions:

(i) $V$ is the vertex set,

(ii) $\gamma: V \rightarrow \mathbb{Z}_{\geq 0}$ is a genus assignment,

(iii) $\pi: V \rightarrow\{0, \infty\}$ is a function, 
(iv) $E$ is the edge set, and

(a) if an edge $e$ connects $v, v^{\prime} \in V$, then $\pi(v) \neq \pi\left(v^{\prime}\right)$, in particular, there are no self edges,

(b) $\Gamma$ has $c$ ordered connected components $\Gamma_{1}, \ldots, \Gamma_{c}$, with vertex and edge sets $\left(V_{i}, E_{i}\right)$ respectively,

(v) $\delta: E \rightarrow \mathbb{Z}_{>0}$ is a degree assignment,

(vi) $N=\{1, \ldots, n\}$ is a set of vertex markings for which each subset $n_{i}$ is incident to $V_{i}$,

(vii) $g_{i}=\sum_{v \in V_{i}} \gamma(v)+h^{1}\left(\Gamma_{i}\right)$,

(viii) $d_{i}=\sum_{e \in E_{i}} \delta(e)$,

(ix) $R^{j}$ is a refinement of $\mu^{j}$ consisting of:

(a) a choice of side $s^{j} \in\{0, \infty\}$,

(b) a distribution of the parts of $\mu^{j}$ to the vertices of $\pi^{-1}\left(s^{j}\right)$ satisfying two properties:

- the parts of $\mu^{j}[i]$ are distributed to the vertices of $\Gamma_{i}$,

- the sum of the parts distributed to $v$ equals the sum of the degrees of edges incident to $v$.

1.3.3. Torus fixed points. Let the moduli point

$$
\left[C \stackrel{f}{\rightarrow} T \stackrel{\epsilon}{\rightarrow} \mathbf{P}^{1}\right] \in \bar{M}_{\mathbf{g}, \mathbf{n}}^{\dagger}\left(\mu^{1}, \ldots, \mu^{m}\right)
$$

be fixed by the torus action. All marked points, nodes, contracted components, and ramification points of $C$ must lie over the torus fixed set $\{0, \infty\}$ of $\mathbf{P}^{1}$. Each irreducible component $D \subset C$ dominant onto $\mathbf{P}^{1}$ must be a Galois cover with full ramification over the two fixed points $\{0, \infty\}$.

We associate a localization graph

$$
\Gamma=\left(V, E, N, \gamma, \pi, \delta,\left(R^{1}, \ldots, R^{m}\right)\right)
$$

to the torus fixed point (4) by the following construction:

(i) Let $V$ be the set of connected components of $(\epsilon \circ f)^{-1}(\{0, \infty\})$.

(ii) Let $\gamma(v)$ be the arithmetic genus of the corresponding component (taken to be 0 if the component is an isolated point).

(iii) Let $\pi(v)$ be the fixed point in $\mathbf{P}^{1}$ over which the corresponding component lies.

(iv) Let $E$ be the set of noncontracted irreducible components $D \subset C$.

(v) Let $\delta(D)$ be the degree of the Galois cover $\left.\epsilon \circ f\right|_{D}$.

(vi) Let $N$ be the marking set.

(ix) Let $R^{j}$ be the refinement of $\mu^{j}$ obtained by the ramification conditions.

All the conditions of a localization graph, including (vii)-(viii), hold by the definition of a stable relative map.

A stack $\bar{M}_{\Gamma}$ together with an action of a finite group $\mathbf{A}_{\Gamma}$ is canonically constructed from $\Gamma$ in Section 1.3.4 below. A canonical inclusion

$$
\tau_{\Gamma} / \mathbf{A}_{\Gamma}: \bar{M}_{\Gamma} / \mathbf{A}_{\Gamma} \rightarrow \bar{M}_{\mathbf{g}, \mathbf{n}}^{\dagger}\left(\mu^{1}, \ldots, \mu^{m}\right)
$$

will be defined. The disjoint union $\bigcup_{\Gamma} \bar{M}_{\Gamma} / \mathbf{A}_{\Gamma}$ will equal the total torus fixed set. 
1.3.4. The torus fixed locus $\bar{M}_{\Gamma} / \mathbf{A}_{\Gamma}$. Let $\Gamma$ be a bipartite graph. The stack $\bar{M}_{\Gamma}$ is defined as a product of auxiliary moduli spaces of curves and maps. The $\mathbf{A}_{\Gamma}$-action is obtained from the automorphisms of $\Gamma$.

Case I. The refinements $\left(R^{1}, \ldots, R^{m}\right)$ lie on both sides 0 and $\infty$.

The data of $\Gamma$ over 0 uniquely defines a moduli space of unparameterized stable relative maps,

$$
\bar{M}_{0}=\bar{M}_{\mathbf{g}_{0}, \mathbf{n}_{0}}\left(\mathbf{R}_{0}, R_{\delta}\right)
$$

where:

(i) $\mathbf{g}_{0}$ is determined by the genera of the vertices $V_{0}=f^{-1}\{0\}$,

(ii) $\mathbf{n}_{0}$ is determined by the markings of $V_{0}$,

(iii) $\mathbf{R}_{0}$ is the set of refinements on side 0 ,

(iv) $R_{\delta}$ is the ramification condition determined by $\delta$.

Let $q_{0}$ denote the new marking associated to $R_{\delta}$.

Similarly, the unparameterized moduli space

$$
\bar{M}_{\infty}=\bar{M}_{\mathbf{g}_{\infty}, \mathbf{n}_{\infty}}\left(R_{\delta}, \mathbf{R}_{\infty}\right)
$$

is defined by the data of $\Gamma$ over $\infty$. Let $q_{\infty}$ denote the new marking associated to $R_{\delta}$. Let $\bar{M}_{\Gamma}=\bar{M}_{0} \times \bar{M}_{\infty}$. The moduli space $\bar{M}_{\Gamma}$ has a virtual class

$$
\left[\bar{M}_{\Gamma}\right]^{\mathrm{vir}}=\left[\bar{M}_{0}\right]^{\mathrm{vir}} \times\left[\bar{M}_{\infty}\right]^{\mathrm{vir}},
$$

determined by the product of the virtual classes of the two factors.

Over $\bar{M}_{\Gamma}$, there is a canonical family of $\mathbb{C}^{*}$-fixed stable relative maps

$$
\pi_{C}: \mathcal{C} \rightarrow \bar{M}_{\Gamma}, \quad \pi_{T}: \mathcal{T} \rightarrow \bar{M}_{\Gamma}, \quad \mathcal{C} \stackrel{f}{\rightarrow} \mathcal{T} \stackrel{\epsilon}{\rightarrow} \mathbf{P}^{1} .
$$

The canonical family is constructed by attaching the universal families over $\bar{M}_{0}$ and $\bar{M}_{\infty}$ according to $\Gamma$. The canonical family yields a canonical morphism of stacks, $\tau_{\Gamma}: \bar{M}_{\Gamma} \rightarrow$ $\bar{M}_{\mathbf{g}, \mathbf{n}}^{\dagger}\left(\mu^{1}, \ldots, \mu^{m}\right)$.

There is a natural automorphism group $\mathbf{A}_{\Gamma}$ acting equivariantly on $\mathcal{C}$ and $\bar{M}_{\Gamma}$ with respect to the morphisms $\rho$ and $\pi_{C}$. The group $\mathbf{A}_{\Gamma}$ acts via automorphisms of the Galois covers (corresponding to the edges) and the symmetries of the graph $\Gamma$. The group $\mathbf{A}_{\Gamma}$ is filtered by an exact sequence of groups,

$$
1 \rightarrow \prod_{e \in E} \mathbb{Z} / \delta(e) \rightarrow \mathbf{A}_{\Gamma} \rightarrow \operatorname{Aut}(\Gamma) \rightarrow 1,
$$

where $\operatorname{Aut}(\Gamma)$ is the automorphism group of $\Gamma: \operatorname{Aut}(\Gamma)$ is the subgroup of the permutation group of the vertices and edges which respects all the structures of $\Gamma$. Aut $(\Gamma)$ naturally acts on $\prod_{e \in E} \mathbb{Z} / \delta(e)$ and $\mathbf{A}_{\Gamma}$ is the semidirect product.

The quotient stack $\bar{M}_{\Gamma} / \mathbf{A}_{\Gamma}$ is a nonsingular Deligne-Mumford stack. The induced map

$$
\tau_{\Gamma} / \mathbf{A}_{\Gamma}: \bar{M}_{\Gamma} / \mathbf{A}_{\Gamma} \rightarrow \bar{M}_{\mathbf{g}, \mathbf{n}}^{\dagger}\left(\mu^{1}, \ldots, \mu^{m}\right)
$$

is a closed immersion of Deligne-Mumford stacks. 
The multiplicity $m(\Gamma)$ and the Euler class of the virtual normal bundle $e\left(N_{\Gamma}^{\mathrm{vir}}\right)$ will be required for the localization formula:

$$
m(\Gamma)=\prod_{e \in E} \delta(e)^{2}, \quad \frac{1}{e\left(N_{\Gamma}^{\mathrm{vir}}\right)}=\frac{1}{t\left(t-\psi_{q_{0}}\right)} \frac{1}{-t\left(-t-\psi_{q_{\infty}}\right)} .
$$

A degenerate configuration occurs over 0 if the following special conditions hold for $\Gamma$ :

(i) all vertices of $V_{0}$ have genus 0 ,

(ii) all vertices of $V_{0}$ have valence 1 ,

(iii) a single $R^{j}$ lies on side 0 and $R^{j}=R_{\delta}$.

Condition (ii) implies that no markings are incident to $V_{0}$.

If $\Gamma$ satisfies (i)-(iii), then $\bar{M}_{0}$ is defined to be a point. The multiplicity formula and Euler class formulas for the degenerate configuration are

$$
m(\Gamma)=\prod_{e \in E} \delta(e), \quad \frac{1}{e\left(N_{\Gamma}^{\mathrm{vir}}\right)}=\frac{1}{t} \frac{1}{-t\left(-t-\psi_{q_{\infty}}\right)} .
$$

Similarly, a degenerate configuration may occur over $\infty$. The treatment is identical with the roles of 0 and $\infty$ interchanged. In fact, degenerate configurations may occur simultaneously at 0 and $\infty$. Then both $\bar{M}_{0}$ and $\bar{M}_{\infty}$ are defined to be points and

$$
m(\Gamma)=1, \quad \frac{1}{e\left(N_{\Gamma}^{\mathrm{vir}}\right)}=\frac{1}{t} \frac{1}{-t} .
$$

Case II. The refinements lie only on side 0 .

The data of $\Gamma$ over 0 defines a moduli space of unparameterized stable relative maps,

$$
\bar{M}_{0}=\bar{M}_{\mathbf{g}_{0}, \mathbf{n}_{0}}\left(\mathbf{R}_{0}, R_{\delta}\right),
$$

as in Case I. The data of $\Gamma$ over $\infty$ determines a product of moduli spaces,

$$
\bar{M}_{\infty}=\prod_{v \in V_{\infty}} \bar{M}_{\gamma(v), \operatorname{val}(v)}
$$

Here, the valence $\operatorname{val}(v)$ of a vertex counts both the incident edges and incident markings. The unstable moduli spaces $\bar{M}_{0,1}$ and $\bar{M}_{0,2}$ arising in the product $(5)$ are viewed as points.

Let $\bar{M}_{\Gamma}$ be the product $\bar{M}_{0} \times \bar{M}_{\infty}$. The moduli space $\bar{M}_{\Gamma}$ has a virtual class

$$
\left[\bar{M}_{\Gamma}\right]^{\mathrm{vir}}=\left[\bar{M}_{0}\right]^{\mathrm{vir}} \times\left[\bar{M}_{\infty}\right],
$$

determined by the product of the virtual class of the first factor and the ordinary fundamental class of the second factor.

There exists a canonical family over $\bar{M}_{\Gamma}$ and a canonical map

$$
\tau_{\Gamma}: \bar{M}_{\Gamma} \rightarrow \bar{M}_{\mathbf{g}, \mathbf{n}}^{\dagger}\left(\mu^{1}, \ldots, \mu^{m}\right)
$$

equivariant with respect to an $\mathbf{A}_{\Gamma}$-action exactly as in Case I. A closed immersion

$$
\tau_{\Gamma} / \mathbf{A}_{\Gamma}: \bar{M}_{\Gamma} / \mathbf{A}_{\Gamma} \rightarrow \bar{M}_{\mathbf{g}, \mathbf{n}}^{\dagger}\left(\mu^{1}, \ldots, \mu^{m}\right)
$$

is obtained. 
We define the multiplicity and the Euler class of the virtual normal bundle of $\bar{M}_{\Gamma}$ in Case II by

$$
m(\Gamma)=\prod_{e \in E} \delta(e), \quad \frac{1}{e\left(N_{\Gamma}^{\mathrm{vir}}\right)}=\frac{1}{t\left(t-\psi_{q_{0}}\right)} \prod_{e \in E} \frac{-t}{(-t)^{\delta(e)} \delta(e) ! / \delta(e)^{\delta(e)}} \prod_{v \in V_{\infty}} \frac{1}{N(v)}
$$

The vertex terms, $N(v)$, are discussed below.

A vertex $v \in V_{\infty}$ is stable if $2 \gamma(v)-2+\operatorname{val}(v)>0$. If $v$ is stable, the moduli space $\bar{M}_{\gamma(v), \operatorname{val}(v)}$ is a factor of $\bar{M}_{\infty}$. The vertex term $N(v)$ is an equivariant cohomology class on the factor $\bar{M}_{\gamma(v), \operatorname{val}(v)}$ in the stable case.

- Let $v \in V_{\infty}$ be a stable vertex. Let $e_{1}, \ldots, e_{l}$ denote the distinct edges incident to $v$ in bijective correspondence to a subset of the local markings of the moduli space $\bar{M}_{\gamma(v), v a l(v)}$. Let $\psi_{i}$ denote the cotangent line of the marking at $v$ corresponding to $e_{i}$, and let $\lambda_{j}$ denote the Chern classes of the Hodge bundle. Then

$$
\frac{1}{N(v)}=\frac{1}{-t} \cdot \prod_{i=1}^{l} \frac{1}{-t / \delta\left(e_{i}\right)-\psi_{i}} \cdot \sum_{j=0}^{\gamma(v)}(-1)^{j} \lambda_{j}(-t)^{\gamma(v)-j} .
$$

If $v \in V_{\infty}$ is an unstable vertex, then $\gamma(v)=0$ and $\operatorname{val}(v) \leq 2$. There are three unstable cases: two with valence 2 and one with valence 1 .

- Let $v \in V_{\infty}$ be an unmarked vertex with $\gamma(v)=0$ and $\operatorname{val}(v)=2$. Let $e_{1}$ and $e_{2}$ be the two incident edges. Then

$$
\frac{1}{N(v)}=\frac{1}{-t} \cdot \frac{1}{-t / \delta\left(e_{1}\right)-t / \delta\left(e_{2}\right)} .
$$

- Let $v \in V_{\infty}$ be a 1 -marked vertex with $\gamma(v)=0$ and $\operatorname{val}(v)=2$. Then

$$
\frac{1}{N(v)}=\frac{1}{-t}
$$

there are no contributing factors.

- Let $v \in V_{\infty}$ be an unmarked vertex with $\gamma(v)=0$ and $\operatorname{val}(v)=1$. Let $e$ be the unique incident edge. Then

$$
\frac{1}{N(v)}=\frac{1}{-t} \cdot \frac{-t}{\delta(e)}
$$

A degenerate configuration may arise over 0 in Case II. The treatment exactly follows the discussion in Case I.

Case III. The refinements lie only on side $\infty$.

The treatment of Case III exactly follows the discussion of Case II with the roles of 0 and $\infty$ interchanged (and $t$ replacing $-t$ in all the formulas). 
1.3.5. The localization formula. The localization formula for the virtual class of the moduli space $\bar{M}_{\mathbf{g}, \mathbf{n}}^{\dagger}\left(\mu^{1}, \ldots, \mu^{m}\right)$ is

$$
\left[\bar{M}_{\mathbf{g}, \mathbf{n}}^{\dagger}\left(\mu^{1}, \ldots, \mu^{m}\right)\right]^{\mathrm{vir}}=\sum_{\Gamma} \frac{m(\Gamma)}{\left|\mathbf{A}_{\Gamma}\right|} \tau_{\Gamma *}\left(\frac{\left[\bar{M}_{\Gamma}\right]^{\mathrm{vir}}}{e\left(N_{\Gamma}^{\mathrm{vir}}\right)}\right),
$$

in localized equivariant Chow theory.

1.3.6. First application. The localization formula immediately yields the following implication.

Lemma 1. Theorem 2(i) is a consequence of Theorem 2(ii).

Proof. Let $\omega$ be the canonical equivariant lift of a relative Gromov-Witten class on the parameterized moduli space $\bar{M}_{\mathbf{g}, \mathbf{n}}^{\dagger}\left(\mu^{1}, \ldots, \mu^{m}\right)$. By the localization formula 6 ,

$$
\rho_{*}\left(\omega \cap\left[\overline{\boldsymbol{M}}_{\mathbf{g}, \mathbf{n}}^{\dagger}\left(\mu^{1}, \ldots, \mu^{m}\right)\right]^{\mathrm{vir}}\right)=\sum_{\Gamma} \frac{m(\Gamma)}{\left|\mathbf{A}_{\Gamma}\right|} \rho_{\Gamma *}\left(\frac{\tau_{\Gamma}^{*}(\omega)}{e\left(N_{\Gamma}^{\mathrm{vir}}\right)} \cap\left[\bar{M}_{\Gamma}\right]^{\mathrm{vir}}\right),
$$

where $\rho_{\Gamma}=\rho \circ \tau_{\Gamma}$.

We now analyze the $\Gamma$ term on the right side of $(7)$. The space $\bar{M}_{\Gamma}$ is a product of unparameterized moduli spaces of stable relative maps and moduli spaces of stable curves. The class $\tau_{\Gamma}^{*}(\omega)$ is composed of Gromov-Witten and tautological classes on these factor spaces (together with powers of $t$ ). Similarly, the expansion of $1 / e\left(N_{\Gamma}^{\mathrm{vir}}\right)$ is composed of Gromov-Witten and tautological classes (together with powers of $t$ ). Hence, by Theorem 2(ii), the equivariant term

$$
\rho_{\Gamma *}\left(\frac{\tau_{\Gamma}^{*}(\omega)}{e\left(N_{\Gamma}^{\mathrm{vir}}\right)} \cap\left[\bar{M}_{\Gamma}\right]^{\mathrm{vir}}\right)
$$

is a series in $t$ with coefficients in the tautological ring of the target of $\rho$.

The nonequivariant limit of the $\rho$ push-forward

$$
\rho_{*}\left(\omega \cap\left[\bar{M}_{\mathbf{g}, \mathbf{n}}^{\dagger}\left(\mu^{1}, \ldots, \mu^{m}\right)\right]^{\mathrm{vir}}\right)
$$

is obtained from the $t^{0}$ coefficient of the right side of 7 .

\section{Theorem 2(ii)}

\subsection{Overview}

We obtain basic relations constraining the $\rho$ push-forward of relative Gromov-Witten classes on the unparameterized spaces from the localization formula on parameterized spaces. The relations are proven to recursively determine all the $\rho$ push-forwards in terms of tautological classes on the target of $\rho$.

For the proof of Theorem 2(ii), we will require relations for the disconnected case. However, for ease of presentation, we first discuss the connected case. The disconnected case follows with minor modifications. 


\subsection{Basic relations I: The connected case}

2.2.1. The set $\Pi(d, n, k)$. Let $d$ and $n$ be integers satisfying $d \geq n>0$. Let $k \geq 0$ be an integer. A partially ordered partition

$$
\bar{\alpha}=\left(\alpha, \alpha^{\prime}\right)
$$

of degree $d$ and order $n$ consists of the following data:

(i) an ordered partition with $n$ (positive) parts of an integer of size at most $d$,

$$
\alpha=\left(\alpha_{1}, \ldots, \alpha_{n}\right), \quad \sum_{i=1}^{n} \alpha_{i} \leq d,
$$

(ii) an unordered partition $\alpha^{\prime}$ (with positive parts) of $d-\sum_{i=1}^{n} \alpha_{i}$.

The partition $\alpha^{\prime}$ may be empty. Let $\ell(\bar{\alpha})$ denote the length of $\bar{\alpha}$,

$$
\ell(\bar{\alpha})=\ell(\alpha)+\ell\left(\alpha^{\prime}\right)=n+\ell\left(\alpha^{\prime}\right) .
$$

Let $\Pi(d, n, k)$ be the set of partially ordered partitions of degree $d$, order $n$, and length at least $d-k$. Our basic relations in the connected case will be indexed by $\Pi(d, n, k)$.

The set $\Pi(d, n, k)$ is stable for $k \geq d-n$ :

$$
\Pi(d, n, k)=\Pi(d, n, d-n) .
$$

Let $\Pi(d, n, \infty)$ denote the stabilized set.

2.2.2. The push-forward construction. Let $g>0$ be the domain genus and let $\bar{\alpha} \in$ $\Pi(d, n, k)$. We will construct a relation

$$
T_{g, \bar{\alpha}}\left(\mu^{1}, \ldots, \mu^{m} \mid \gamma\right)
$$

where:

(i) $\mu^{1}, \ldots, \mu^{m}$ are partitions of $d$,

(ii) $\gamma$ is a monomial $\prod_{j=1}^{n^{\prime}} \psi_{p_{j}}^{r_{j}} \cup \prod_{j=1}^{m} \psi_{q_{j}}^{s_{j}}$.

The relation $T$ will be obtained from equivariant localization on

$$
\bar{M}_{g, n+\ell\left(\alpha^{\prime \prime}\right)+n^{\prime}}^{\dagger}\left(\mu^{1}, \ldots, \mu^{m}\right)
$$

where $\alpha^{\prime \prime}$ is the (possibly empty) subpartition of $\alpha^{\prime}$ consisting of parts of size at least 2 . We will use the abbreviated notation $\bar{M}^{\dagger}$ for the moduli space of stable relative maps 8 .

The moduli space $\bar{M}^{\dagger}$ carries $n+\ell\left(\alpha^{\prime \prime}\right)+n^{\prime}$ markings of type $p$ indexed by the following conventions:

(i) let $p_{i}$ denote the first $n$,

(ii) let $p_{i^{\prime \prime}}$ denote the middle $\ell\left(\alpha^{\prime \prime}\right)$,

(iii) let $p_{j}$ denote the last $n^{\prime}$.

Let the middle $\ell\left(\alpha^{\prime \prime}\right)$ markings be placed in correspondence with the parts of $\alpha^{\prime \prime}$. 
Define the equivariant relative Gromov-Witten class $\omega$ on the moduli space $\bar{M}^{\dagger}$ as a product of four factors:

$$
\omega=\prod_{i=1}^{n} \psi_{p_{i}}^{\alpha_{i}-1} \operatorname{ev}_{p_{i}}^{*}([\infty]) \cdot \prod_{i^{\prime \prime}=1}^{\ell\left(\alpha^{\prime \prime}\right)} \psi_{p_{i^{\prime \prime}}}^{\alpha_{i^{\prime \prime}}^{\prime \prime}-1} \operatorname{ev}_{p_{i^{\prime \prime}}}^{*}([\infty]) \cdot \gamma \cdot\left(\operatorname{ev}_{q_{1}}^{*}([0])\right)^{2+\ell(\bar{\alpha})-d+k}
$$

where $\gamma$ is the monomial in the argument of $T$. The degree of $\omega$ is

$$
k+n+\ell\left(\alpha^{\prime \prime}\right)+\sum_{j=1}^{n^{\prime}} r_{j}+\sum_{j=1}^{m} s_{j}+2
$$

Since the virtual dimension of $\bar{M}^{\dagger}$ is

$$
2 g-2+2 d+n+\ell\left(\alpha^{\prime \prime}\right)+n^{\prime}+\sum_{i=1}^{m}\left(1+l\left(\mu_{i}\right)-d\right),
$$

the dimension of $\omega \cap\left[\bar{M}^{\dagger}\right]^{\mathrm{vir}}$ is

$$
2 g-2+2 d+n^{\prime}+\sum_{i=1}^{m}\left(1+l\left(\mu_{i}\right)-d\right)-\left(k+\sum_{j=1}^{n^{\prime}} r_{j}+\sum_{j=1}^{m} s_{j}+2\right) .
$$

The moduli space $\bar{M}^{\dagger}$ carries $n+\ell\left(\alpha^{\prime \prime}\right)+n^{\prime}+\sum_{i=1}^{m} \ell\left(\mu^{i}\right)$ total domain markings. Let

$$
\rho^{\prime \prime}: \bar{M}^{\dagger} \rightarrow \bar{M}_{n+n^{\prime}+\sum_{i=1}^{m} \ell\left(\mu^{i}\right)}
$$

be the stabilization map obtained from $\rho$ after forgetting the markings corresponding to $\alpha^{\prime \prime}$. Since the class $\mathrm{ev}_{q_{1}}^{*}([0])$ occurs in $\omega$ with total exponent at least 2 , the nonequivariant limit of $\omega$ is 0 . Hence, in the nonequivariant limit, the push-forward $\rho_{*}^{\prime \prime}\left(\omega \cap\left[\bar{M}^{\dagger}\right]^{\text {vir }}\right)$ vanishes:

$$
\rho_{*}^{\prime \prime}\left(\omega \cap\left[\bar{M}^{\dagger}\right]^{\mathrm{vir}}\right)=0 \in A_{*}\left(\bar{M}_{g, n+n^{\prime}+\sum_{i=1}^{m} \ell\left(\mu^{i}\right)}\right) .
$$

The left side of (9) can be calculated by the localization formula (7). As $\omega$ is a nontrivial equivariant class, equation (97) yields a nontrivial relation after localizing and taking the nonequivariant limit,

$$
T_{g, \bar{\alpha}}\left(\mu^{1}, \ldots, \mu^{m} \mid \gamma\right)=\sum_{\Gamma} \frac{m(\Gamma)}{\left|\mathbf{A}_{\Gamma}\right|} \rho_{\Gamma *}^{\prime \prime}\left(\frac{\tau_{\Gamma}^{*}(\omega)}{e\left(N_{\Gamma}^{\mathrm{vir}}\right)} \cap\left[\bar{M}_{\Gamma}\right]^{\mathrm{vir}}\right)=0,
$$

where $\rho_{\Gamma}^{\prime \prime}=\rho^{\prime \prime} \circ \tau_{\Gamma}$.

2.2.3. The principal terms of $T$. A localization graph $\Gamma$ corresponding to a fixed locus of $\bar{M}^{\dagger}$ is of type $\bar{\beta} \in \Pi(d, n, k)$ if the following properties are satisfied:

(i) the vertex set $V_{0}$ consists of a single vertex $v_{0}$ of genus $g$,

(ii) the edge set $E$ is in bijective correspondence to the $\ell(\bar{\beta})$ parts of $\bar{\beta}$, 
(iii) the vertex set $V_{\infty}$ consists of $\ell(\bar{\beta})$ vertices each incident to a unique edge,

(iv) the $n+\ell\left(\alpha^{\prime \prime}\right)+n^{\prime}$ markings of type $p$ are distributed by the following rules:

(a) the first $n$ markings lie over $\infty$ with the $i$ th marking incident to the vertex corresponding to the $i$ th part of the first partition $\beta$ of $\bar{\beta}$,

(b) the second $\ell\left(\alpha^{\prime \prime}\right)$ markings lie over $\infty$ on distinct vertices incident to the edges corresponding to the parts of the second partition $\beta^{\prime}$ of $\bar{\beta}$,

(c) the third $n^{\prime}$ markings are all incident to $v_{0}$,

(v) all refinements lie on side 0 .

Let $\Gamma_{\bar{\beta}}$ denote the set of localization graphs of type $\bar{\beta}$. Since $g>0$, the localization graphs $\Gamma \in \Gamma_{\bar{\beta}}$ are never degenerate over 0 .

The principal terms of the relation $T$ are indexed by partially ordered partitions $\bar{\beta} \in$ $\Pi(d, n, k)$. The principal term of $T$ of type $\bar{\beta}$ is

$$
T_{g, \bar{\alpha}}\left(\mu^{1}, \ldots, \mu^{m} \mid \gamma\right)[\bar{\beta}]=\sum_{\Gamma \in \Gamma_{\bar{\beta}}} \frac{m(\Gamma)}{\left|\mathbf{A}_{\Gamma}\right|} \rho_{\Gamma *}^{\prime \prime}\left(\frac{\tau_{\Gamma}^{*}(\omega)}{e\left(N_{\Gamma}^{\mathrm{vir}}\right)} \cap\left[\bar{M}_{\Gamma}\right]^{\mathrm{vir}}\right) .
$$

We may compute the principal term of $T_{g, \bar{\alpha}}\left(\mu^{1}, \ldots, \mu^{m} \mid \gamma\right)$ of type $\bar{\beta}$ explicitly. However, we must define a function $S\left[\alpha^{\prime \prime}\right]\left(\beta^{\prime}\right)$ which arises naturally from the localization formula.

Let $\alpha^{\prime \prime}$ and $\beta^{\prime}$ be two unordered partitions. Select an ordering of the parts,

$$
\alpha^{\prime \prime}=\left(\alpha_{1}^{\prime \prime}, \ldots, \alpha_{\ell\left(\alpha^{\prime \prime}\right)}^{\prime \prime}\right), \quad \beta^{\prime}=\left(\beta_{1}^{\prime}, \ldots, \beta_{\ell\left(\beta^{\prime}\right)}^{\prime}\right) .
$$

Define the integer sets $\underline{0}=\emptyset$ and $\underline{i}=\{1, \ldots, i\}$ for each $i \geq 1$. Define the function $S\left[\alpha^{\prime \prime}\right]\left(\beta^{\prime}\right)$ by the following rules:

(i) if $\ell\left(\alpha^{\prime \prime}\right)>\ell\left(\beta^{\prime}\right)$, then $S\left[\alpha^{\prime \prime}\right]\left(\beta^{\prime}\right)=0$,

(ii) if $\ell\left(\alpha^{\prime \prime}\right) \leq \ell\left(\beta^{\prime}\right)$, then

$$
S\left[\alpha^{\prime \prime}\right]\left(\beta^{\prime}\right)=\sum_{l: \ell\left(\alpha^{\prime \prime}\right) \rightarrow \underline{\ell\left(\beta^{\prime}\right)}} \prod_{j=1}^{\ell\left(\alpha^{\prime \prime}\right)} \frac{1}{\left(\beta_{l(j)}^{\prime}\right)^{\alpha_{j}^{\prime \prime}-1}} \cdot \prod_{i \notin \operatorname{Im}(l)} \frac{1}{\beta_{i}^{\prime}} .
$$

is a sum over all injections $\iota: \underline{\ell\left(\alpha^{\prime \prime}\right)} \rightarrow \underline{\ell\left(\beta^{\prime}\right)}$.

The function $S\left[\alpha^{\prime \prime}\right]\left(\beta^{\prime}\right)$ depends only upon the unordered partitions $\alpha^{\prime \prime}$ and $\beta^{\prime}$.

Let $\bar{M}(\bar{\beta})$ denote the moduli space of unparameterized maps,

$$
\bar{M}(\bar{\beta})=\bar{M}_{g, n^{\prime}}\left(\bar{\beta}, \mu^{1}, \ldots, \mu^{m}\right),
$$

with markings of type $q$ indexed by $0,1, \ldots, m$. Define the standard Gromov-Witten class $\omega_{\bar{\beta}}$ on the moduli space $\bar{M}(\bar{\beta})$ by

$$
\omega_{\bar{\beta}}=\gamma \cdot \psi_{q_{0}}^{\ell(\bar{\beta})-d+k} .
$$

The degree of $\omega_{\bar{\beta}}$ is

$$
\sum_{j=1}^{n^{\prime}} r_{j}+\sum_{j=1}^{m} s_{j}+\ell(\bar{\beta})-d+k .
$$


Since the dimension of $\bar{M}(\bar{\beta})$ is

$$
2 g-2+2 d+n^{\prime}+(1+\ell(\bar{\beta})-d)+\sum_{j=1}^{m}\left(1+\ell\left(\mu^{j}\right)-d\right)-3,
$$

the dimension of $\omega_{\bar{\beta}} \cap[\bar{M}(\bar{\beta})]^{\mathrm{vir}}$ is

$$
2 g-2+2 d+n^{\prime}+\sum_{i=1}^{m}\left(1+l\left(\mu_{i}\right)-d\right)-\left(k+\sum_{j=1}^{n^{\prime}} r_{j}+\sum_{j=1}^{m} s_{j}+2\right),
$$

equal to the dimension of $\omega \cap\left[\bar{M}^{\dagger}\right]^{\text {vir }}$.

A direct application of the localization formula (7) yields the following result.

Lemma 2. The principal term of $T_{g, \bar{\alpha}}\left(\mu^{1}, \ldots, \mu^{m} \mid \gamma\right)$ of type $\bar{\beta}$ is

$$
\left(\prod_{i=1}^{n} \frac{1}{\beta_{i}^{\alpha_{i}-1}} S\left[\alpha^{\prime \prime}\right]\left(\beta^{\prime}\right)(-1)^{n+\ell\left(\beta^{\prime}\right)} \eta(\bar{\beta})\right) \cdot \rho_{*}^{\prime \prime}\left(\omega_{\bar{\beta}} \cap[\bar{M}(\bar{\beta})]^{\mathrm{vir}}\right),
$$

where $\eta(\bar{\beta})$ is the nonvanishing factor

$$
\frac{1}{\left|\operatorname{Aut}\left(\beta^{\prime}\right)\right|} \prod_{i=1}^{n} \frac{1}{(-1)^{\beta_{i}} \beta_{i} ! / \beta_{i}^{\beta_{i}}} \prod_{i=1}^{\ell\left(\beta^{\prime}\right)} \frac{1}{(-1)^{\beta_{i}^{\prime}} \beta_{i}^{\prime} ! / \beta_{i}^{\prime \beta_{i}^{\prime}}} .
$$

2.3. Basic relations II: The disconnected case

2.3.1. The set $\Pi(\mathbf{d}, \mathbf{n}, k)$. Let $\mathbf{d}=\left(d_{1}, \ldots, d_{c}\right)$ be an ordered degree partition, and let

$$
d=\sum_{i=1}^{c} d_{i}
$$

Let $\mathbf{n}=\left(n_{1}, \ldots, n_{c}\right)$ be an ordered set partition of $\underline{n}$ for which

$$
d_{i} \geq\left|n_{i}\right|>0
$$

for all $i$. Let $k \geq 0$ be an integer. A partially ordered partition

$$
\bar{\alpha}=\left(\left(\alpha[1], \alpha^{\prime}[1]\right), \ldots,\left(\alpha[c], \alpha^{\prime}[c]\right)\right)
$$

of degree $\mathbf{d}$ and order $\mathbf{n}$ consists of the following data:

(i) ordered partitions with $\left|n_{i}\right|$ (positive) parts of integers of size at most $d_{i}$,

$$
\alpha[i]=\left(\alpha[i]_{1}, \ldots, \alpha[i]_{\left|n_{i}\right|}\right), \quad \sum_{j=1}^{n} \alpha[i]_{j} \leq d_{i}
$$

(ii) unordered partitions $\alpha^{\prime}[i]$ (with positive parts) of $d_{i}-\sum_{j=1}^{n} \alpha[i]_{j}$. 
The partitions $\alpha^{\prime}[i]$ may be empty. Let $\ell(\bar{\alpha})$ denote the length of $\bar{\alpha}$,

$$
\ell(\bar{\alpha})=\sum_{i=1}^{c}\left(\ell(\alpha[i])+\ell\left(\alpha^{\prime}[i]\right)\right)=n+\sum_{i=1}^{c} \ell\left(\alpha^{\prime}[i]\right) .
$$

Let $\Pi(\mathbf{d}, \mathbf{n}, k)$ be the set of partially ordered partitions of degree $\mathbf{d}$, order $\mathbf{n}$, and length at least $d-k$. Our basic relations in the disconnected case will be indexed by $\Pi(\mathbf{d}, \mathbf{n}, k)$.

The set $\Pi(\mathbf{d}, \mathbf{n}, k)$ is stable for $k \geq d-n$ :

$$
\Pi(\mathbf{d}, \mathbf{n}, k)=\Pi(\mathbf{d}, \mathbf{n}, d-n) .
$$

Let $\Pi(\mathbf{d}, \mathbf{n}, \infty)$ denote the stabilized set.

2.3.2. The push-forward construction. Let $\mathbf{g}=\left(g_{1}, \ldots, g_{c}\right)$ be an ordered set of genera where not all $g_{i}$ are 0 . Let $\bar{\alpha} \in \Pi(\mathbf{d}, \mathbf{n}, k)$. We will construct a relation

$$
T_{\mathbf{g}, \bar{\alpha}}\left(\mu^{1}, \ldots, \mu^{m} \mid \gamma\right) \text {, }
$$

where:

(i) $\mu^{1}, \ldots, \mu^{m}$ are partitions of type $\mathbf{d}$,

(ii) $\gamma$ is a monomial $\prod_{j=1}^{n^{\prime}} \psi_{p_{j}}^{r_{j}} \cup \prod_{j=1}^{m} \psi_{q_{j}}^{s_{j}}$.

The relation $T$ will be obtained from equivariant localization on

$$
\bar{M}_{\mathbf{g}, \mathbf{n}+\ell\left(\alpha^{\prime \prime}\right)+\mathbf{n}^{\prime}}^{\dagger}\left(\mu^{1}, \ldots, \mu^{m}\right),
$$

where $\alpha^{\prime \prime}$ is the (possibly empty) subpartition of $\left(\alpha^{\prime}[1], \ldots, \alpha^{\prime}[c]\right)$ consisting of parts of size at least 2. The last $n^{\prime}$ markings of type $p$ are distributed on the domain components by $\mathbf{n}^{\prime}$. As before, let $\bar{M}^{\dagger}$ denote the moduli space of stable relative maps 10 .

Define the equivariant relative Gromov-Witten class $\omega$ on the moduli space $\bar{M}^{\dagger}$ as a product of four factors:

$$
\begin{aligned}
\omega= & \prod_{i=1}^{c}\left(\prod_{j=1}^{\left|n_{i}\right|} \psi_{p_{j}}^{\alpha[i]_{j}-1} \operatorname{ev}_{p_{j}}^{*}([\infty]) \cdot \prod_{j^{\prime \prime}=1}^{\ell\left(\alpha^{\prime \prime}[i]\right)} \psi_{p_{j^{\prime \prime}}}^{\alpha^{\prime \prime}\left[i_{j^{\prime \prime}}-1\right.} \operatorname{ev}_{p_{j^{\prime \prime}}}^{*}([\infty])\right) \\
& \cdot \gamma \cdot\left(\mathrm{ev}_{q_{1}}^{*}([0])\right)^{2+\ell(\bar{\alpha})-d+k},
\end{aligned}
$$

where $\gamma$ is the monomial in the argument of $T$.

The dimension calculus proceeds exactly as in the connected case (replacing the connected genus $g$ by the arithmetic genus $\sum_{i=1}^{c} g_{i}-c+1$ in the disconnected case).

The moduli space $\bar{M}^{\dagger}$ carries $n+\ell\left(\alpha^{\prime \prime}\right)+n^{\prime}+\sum_{i=1}^{m} \ell\left(\mu^{i}\right)$ total domain markings. Let

$$
\rho^{\prime \prime}: \bar{M}^{\dagger} \rightarrow \prod_{i=1}^{c} \bar{M}_{g_{i},\left|n_{i}\right|+\left|n_{i}^{\prime}\right|+\sum_{j=1}^{m} \ell\left(\mu^{j}[i]\right)}
$$

be the stabilization map obtained from $\rho$ after forgetting the markings corresponding to $\alpha^{\prime \prime}$. As before, the push-forward vanishes in the nonequivariant limit,

$$
\rho_{*}^{\prime \prime}\left(\omega \cap\left[\bar{M}^{\dagger}\right]^{\mathrm{vir}}\right)=0 .
$$


The left side of (12) can be calculated by the localization formula (7) to yield the relation

$$
T_{\mathbf{g}, \bar{\alpha}}\left(\mu^{1}, \ldots, \mu^{m} \mid \gamma\right)=\sum_{\Gamma} \frac{m(\Gamma)}{\left|\mathbf{A}_{\Gamma}\right|} \rho_{\Gamma *}^{\prime \prime}\left(\frac{\tau_{\Gamma}^{*}(\omega)}{e\left(N_{\Gamma}^{\text {vir }}\right)} \cap\left[\bar{M}_{\Gamma}\right]^{\mathrm{vir}}\right)=0 .
$$

2.3.3. The principal terms of $T$. A localization graph $\Gamma$ corresponding to a fixed locus of $\bar{M}^{\dagger}$ is of type $\bar{\beta} \in \Pi(\mathbf{d}, \mathbf{n}, k)$ if the following properties are satisfied:

(i) the vertex set $V_{0}$ consists of vertices $v_{1,0}, \ldots, v_{c, 0}$ with genus assignments $g_{1}, \ldots, g_{c}$ respectively,

(ii) the edge set $E$ is in bijective correspondence to the $\ell(\bar{\beta})$ parts of $\bar{\beta}$,

(iii) the vertex $v_{i, 0}$ is incident to the edges corresponding to the parts of $\left(\beta[i], \beta^{\prime}[i]\right)$,

(iv) the vertex set $V_{\infty}$ consists of $\ell(\bar{\beta})$ vertices each incident to a unique edge,

(v) the $n+\ell\left(\alpha^{\prime \prime}\right)+n^{\prime}$ markings of type $p$ are distributed by the following rules:

(a) the markings $n_{i}$ lie over $\infty$ with the $j$ th marking incident to the vertex corresponding to the $j$ th part of $\beta[i]$,

(b) the middle $\ell\left(\alpha^{\prime \prime}[i]\right)$ markings lie over $\infty$ on distinct vertices incident to the edges corresponding to the parts of $\beta^{\prime}[i]$,

(c) the markings $n_{i}^{\prime}$ are incident to $v_{i, 0}$,

(vi) all refinements lie on side 0 .

Let $\Gamma_{\bar{\beta}}$ denote the set of localization graphs of type $\bar{\beta}$. Since not all $g_{i}$ are 0 , the localization graphs $\Gamma \in \Gamma_{\bar{\beta}}$ are never degenerate over 0 .

The principal terms of the relation $T$ are indexed by partially ordered partitions $\bar{\beta} \in$ $\Pi(\mathbf{d}, \mathbf{n}, k)$. The principal term of $T$ of type $\bar{\beta}$ is

$$
T_{\mathbf{g}, \bar{\alpha}}\left(\mu^{1}, \ldots, \mu^{m} \mid \gamma\right)[\bar{\beta}]=\sum_{\Gamma \in \Gamma_{\bar{\beta}}} \frac{m(\Gamma)}{\left|\mathbf{A}_{\Gamma}\right|} \rho_{\Gamma *}^{\prime \prime}\left(\frac{\tau_{\Gamma}^{*}(\omega)}{e\left(N_{\Gamma}^{\mathrm{vir}}\right)} \cap\left[\bar{M}_{\Gamma}\right]^{\mathrm{vir}}\right) .
$$

The principal term of $T_{\mathbf{g}, \bar{\alpha}}\left(\mu^{1}, \ldots, \mu^{m} \mid \gamma\right)$ of type $\bar{\beta}$ may also be explicitly computed from the localization formula.

Let $\bar{M}(\bar{\beta})$ denote the moduli space of unparameterized maps,

$$
\bar{M}(\bar{\beta})=\bar{M}_{\mathbf{g}, \mathbf{n}^{\prime}}\left(\bar{\beta}, \mu^{1}, \ldots, \mu^{m}\right),
$$

with markings of type $q$ indexed by $0,1, \ldots, m$. Define the standard Gromov-Witten class $\omega_{\bar{\beta}}$ on the moduli space $\bar{M}(\bar{\beta})$ by

$$
\omega_{\bar{\beta}}=\gamma \cdot \psi_{q_{0}}^{\ell(\bar{\beta})-d+k} .
$$

A direct application of the localization formula (7) yields the following result.

Lemma 3. The principal term of $T_{\mathbf{g}, \bar{\alpha}}\left(\mu^{1}, \ldots, \mu^{m} \mid \gamma\right)$ of type $\bar{\beta}$ is

$$
\begin{array}{r}
\prod_{i=1}^{c}\left(\prod_{j=1}^{\left|n_{i}\right|} \frac{1}{\left(\beta[i]_{j}\right)^{\alpha[i]_{j}-1}} S\left[\alpha^{\prime \prime}[i]\right]\left(\beta^{\prime}[i]\right)(-1)^{\left|n_{i}\right|+\ell\left(\beta^{\prime}[i]\right)} \eta\left(\left(\beta[i], \beta^{\prime}[i]\right)\right)\right) \\
\cdot \rho_{*}^{\prime \prime}\left(\omega_{\bar{\beta}} \cap[\bar{M}(\bar{\beta})]^{\mathrm{vir}}\right) .
\end{array}
$$




\subsection{The matrix $\mathbf{M}$}

Let $\mathbf{M}(\mathbf{d}, \mathbf{n}, k)$ be the matrix with rows and columns indexed by the set $\Pi(\mathbf{d}, \mathbf{n}, k)$ defined by the prefactors of the principal terms of the basic relations. For $\bar{\alpha}, \bar{\beta} \in \Pi(\mathbf{d}, \mathbf{n}, k)$, let the element of $\mathbf{M}(\mathbf{d}, \mathbf{n}, k)$ in position $(\bar{\alpha}, \bar{\beta})$ be

$$
\prod_{i=1}^{c}\left(\prod_{j=1}^{\left|n_{i}\right|} \frac{1}{\left(\beta[i]_{j}\right)^{\alpha[i]_{j}-1}} S\left[\alpha^{\prime \prime}[i]\right]\left(\beta^{\prime}[i]\right)(-1)^{\left|n_{i}\right|+\ell\left(\beta^{\prime}[i]\right)} \eta\left(\left(\beta[i], \beta^{\prime}[i]\right)\right)\right),
$$

the prefactor of the principal term of $T_{\mathbf{g}, \bar{\alpha}}\left(\mu^{1}, \ldots, \mu^{m} \mid \gamma\right)$ of type $\bar{\beta}$.

Lemma 4. $\mathbf{M}(\mathbf{d}, \mathbf{n}, k)$ is invertible.

Lemma 4 will be proven in Section 3 . The nonsingularity of $\mathbf{M}(\mathbf{d}, \mathbf{n}, k)$ plays a fundamental role in our proof of Theorem 2(ii).

\subsection{Proof of Theorem 2(ii)}

2.5.1. Overview. Let $\bar{M}_{\mathbf{g}, \mathbf{n}^{\prime}}\left(\mu^{1}, \ldots, \mu^{m}\right)$ be a moduli space of stable relative maps with $c$ domain components, degree partition $\mathbf{d}$, and total degree $d$. Let $\omega$ be a relative GromovWitten class on $\bar{M}_{\mathbf{g}, \mathbf{n}^{\prime}}\left(\mu^{1}, \ldots, \mu^{m}\right)$. Let $\rho_{*}\left(\omega \cap[\bar{M}]^{\text {vir }}\right)$ denote the push-forward

$$
\rho_{*}\left(\omega \cap\left[\bar{M}_{\mathbf{g}, \mathbf{n}^{\prime}}\left(\mu^{1}, \ldots, \mu^{m}\right)\right]^{\mathrm{vir}}\right) .
$$

We must prove $\rho_{*}\left(\omega \cap[\bar{M}]^{\mathrm{vir}}\right)$ is a tautological class on the target of $\rho$.

The proof consists of a multilayer induction using the basic relations $T$. We will prove the result for each total degree $d$ separately. The proof will follow the notation of Section 2.3

2.5.2. Genus induction. Since all algebraic cycle classes on products of moduli spaces of genus 0 pointed curves are tautological $[\overline{\mathrm{K}}]$, the class $\rho_{*}\left(\omega \cap[\bar{M}]^{\mathrm{vir}}\right)$ is certainly tautological if all the domain genera $g_{i}$ are 0 .

We will proceed by induction on the arithmetic genus

$$
g(\mathbf{g})=\sum_{i=1}^{c} g_{i}-c+1
$$

of the domain curve. The smallest value for the arithmetic genus is $-d+1$, which occurs when the number of domain components equals $d$ and $\mathbf{g}=(0, \ldots, 0), \mathbf{d}=(1, \ldots, 1)$. The base case of the genus induction holds as all domain genera in the base case are 0 .

2.5.3. Induction on the markings. We now induct upon the number of markings $m$ of type $q$. We first establish the base case $m=2$. In fact, all aspects of the proof of the full result are manifest in the base case. We will also induct on the number $n^{\prime}$ of markings of type $p$. The induction on type $p$ markings is straightforward and plays a very minor role. 
Let $\mathbf{g}$ be a genus distribution with not all genera 0 , and let $\mathbf{d}$ be a degree distribution. Let a partition $\mu^{1}$ of type $\mathbf{d}$ and a relative Gromov-Witten class

$$
\gamma=\prod_{j=1}^{n^{\prime}} \psi_{p_{j}}^{r_{j}} \cup \psi_{q_{1}}^{s_{1}}
$$

be given. Let $\mathbf{n}$ have cardinalities $|\mathbf{n}|=(1, \ldots, 1)$. We will first consider all basic relations

$$
T_{\mathbf{g}, \bar{\alpha}}\left(\mu^{1} \mid \gamma\right)
$$

where $\bar{\alpha} \in \Pi(\mathbf{d}, \mathbf{n}, k)$. Let us analyze the terms of the relations 13 . Let $\Gamma$ be a localization graph. Since $\mathrm{ev}_{p_{j}}^{*}([\infty])$ occurs in 11 for all the first $n+\ell\left(\alpha^{\prime \prime}\right)$ markings of type $p$, only graphs $\Gamma$ for which these markings lie over $\infty$ contribute nontrivially to the basic relations. Similarly, since $\mathrm{ev}_{q_{1}}^{*}([0])$ occurs in $\left[11\right.$, only graphs $\Gamma$ for which $R^{1}$ lies on side 0 contribute.

As there are no refinements on side $\infty$, we are in Case II of the localization formula. We find $\bar{M}_{\Gamma}=\bar{M}_{0} \times \bar{M}_{\infty}$, where

$$
\bar{M}_{0}=\bar{M}_{\mathbf{g}_{0}, \mathbf{n}_{0}^{\prime}}\left(R^{1}, R_{\delta}\right)
$$

and $\bar{M}_{\infty}$ is simply a product of moduli spaces of pointed curves. If

$$
g\left(\mathbf{g}_{0}\right)<g(\mathbf{g}) \text { or } n_{0}^{\prime}<n^{\prime},
$$

then the localization terms over 0 of $\Gamma$ push forward under $\rho^{\prime \prime}$ to tautological classes by the induction hypotheses on the genus and the marking number $n^{\prime}$. The localization terms over $\infty$ certainly push forward to tautological classes.

The graphs $\Gamma$ satisfying $g\left(\mathbf{g}_{0}\right)=g(\mathbf{g})$ have only genus 0 , edge valence 1 vertices over $\infty$. If $n_{0}^{\prime}=n^{\prime}$, only the first $n+\ell\left(\alpha^{\prime \prime}\right)$ markings of type $p$ lie over $\infty$. Of these, the first $n$ markings are distributed on the different connected components of $\Gamma$ since $|\mathbf{n}|=(1, \ldots, 1)$. Since all the parts of $\alpha^{\prime \prime}$ are at least two, the second $\ell\left(\alpha^{\prime \prime}\right)$ markings of type $p$ trivialize the contribution of $\Gamma$ if any vertex of marking valence greater than 1 occurs over $\infty$. An ordered partition $\bar{\beta}$ is obtained from $\Gamma$ from the edge degrees. By dimension considerations, the contribution of $\Gamma$ is trivial unless $\bar{\beta} \in \Pi(\mathbf{d}, \mathbf{n}, k)$.

We have proven that for contributing graphs $\Gamma$, either condition 14 holds or $\Gamma \in \Gamma_{\bar{\beta}}$. Therefore, the nonprincipal terms of the basic relations 13 all push forward via $\rho^{\prime \prime}$ to tautological classes.

The principal terms of the basic relations (13) taken for all $\bar{\alpha} \in \Pi(\mathbf{d}, \mathbf{n}, k)$ determine a nonsingular matrix of prefactors by Lemmas 3 and 4 . We conclude that, for all $\bar{\beta} \in$ $\Pi(\mathbf{d}, \mathbf{n}, k)$,

$$
\rho_{*}^{\prime \prime}\left(\gamma \cdot \psi_{q_{0}}^{\ell(\bar{\beta})-d+k} \cap\left[\bar{M}_{\mathbf{g}, \mathbf{n}^{\prime}}\left(\bar{\beta}, \mu^{1}\right)\right]^{\mathrm{vir}}\right)
$$

is tautological in the target moduli of curves. By considering the classes 15 for all $k \geq 0$, we find,

$$
\rho_{*}^{\prime \prime}\left(\omega \cap\left[\bar{M}_{\mathbf{g}, \mathbf{n}^{\prime}}\left(\bar{\beta}, \mu^{1}\right)\right]^{\mathrm{vir}}\right)
$$

is tautological on the target for all relative Gromov-Witten classes $\omega$ and $\bar{\beta} \in \Pi(\mathbf{d}, \mathbf{n}, \infty)$. We have exhausted the basic relations $[13$ for the element $|\mathbf{n}|=(1, \ldots, 1)$. 
If $\mathbf{d}=(1, \ldots, 1)$, we have completed the $m=2$ induction base since $\rho=\rho^{\prime \prime}$. However, if $\mathbf{d}$ has parts of size greater than 1 , then $\rho \neq \rho^{\prime \prime}$ for general $\bar{\beta}$. To proceed, consider an augmentation

$$
|\mathbf{n}|=(1, \ldots, 1,2,1, \ldots, 1)
$$

subject to $d_{i} \geq\left|n_{i}\right|>0$. We will exhaust the basic relations for the augmented $\mathbf{n}$. We will repeat the cycle of augmentation and exhaustion until all $\mathbf{n}$ satisfying $d_{i} \geq\left|n_{i}\right|>0$ have been considered.

We repeat the entire argument for the augmented $\mathbf{n}$. The argument is identical except for one important difference. For graphs $\Gamma$ satisfying

$$
g\left(\mathbf{g}_{0}\right)=g(\mathbf{g}), \quad n_{0}^{\prime}=n^{\prime},
$$

the first $n$ markings of type $p$ may not be incident to distinct vertices over $\infty$. However, the graphs which contain common incidences yield tautological push-forwards by the analysis for lower $\mathbf{n}$. After each cycle of the analysis, we conclude that

$$
\rho_{*}^{\prime \prime}\left(\omega \cap\left[\bar{M}_{\mathbf{g}, \mathbf{n}^{\prime}}\left(\bar{\beta}, \mu^{1}\right)\right]^{\mathrm{vir}}\right)
$$

is tautological in the target for all relative Gromov-Witten classes $\omega$ and $\bar{\beta} \in \Pi(\mathbf{d}, \mathbf{n}, \infty)$. The $m=2$ base case is proven.

2.5.4. The full induction. The full induction exactly follows the argument for the $m=2$ base case. Let $m \geq 3$.

Let $\mathbf{g}$ be a genus distribution with not all genera 0 , and let $\mathbf{d}$ be a degree distribution. Let partitions $\mu^{1}, \ldots, \mu^{m-1}$ of type $\mathbf{d}$ and a relative Gromov-Witten class

$$
\gamma=\prod_{j=1}^{n^{\prime}} \psi_{p_{j}}^{r_{j}} \cup \prod_{j=1}^{m-1} \psi_{q_{j}}^{s_{j}}
$$

be given. We consider the basic relations

$$
T_{\mathbf{g}, \bar{\alpha}}\left(\mu^{1}, \ldots, \mu^{m-1} \mid \gamma\right),
$$

where $\bar{\alpha} \in \Pi(\mathbf{d}, \mathbf{n}, k)$.

Let $\Gamma$ be a localization graph. As before, only graphs $\Gamma$ for which the first $n+$ $\ell\left(\alpha^{\prime \prime}\right)$ markings of type $p$ lie over $\infty$ contribute nontrivially to the basic relations. Since $\mathrm{ev}_{q_{1}}^{*}([0])$ occurs in $(11)$, only graphs $\Gamma$ for which $R^{1}$ lies on side 0 contribute.

If there are refinements over $\infty$, then we are in Case I of the localization formula. We find $\bar{M}_{\Gamma}=\bar{M}_{0} \times \bar{M}_{\infty}$, where

$$
\bar{M}_{0}=\bar{M}_{\mathbf{g}_{0}, \mathbf{n}_{0}^{\prime}}\left(\mathbf{R}_{0}, R_{\delta}\right), \quad \bar{M}_{\infty}=\bar{M}_{\mathbf{g}_{0}, \mathbf{n}_{0}^{\prime}}\left(R_{\delta}, \mathbf{R}_{\infty}\right) .
$$

Since the graph $\Gamma$ has at most $m-2$ refinements on side $\infty$, both $\bar{M}_{0}$ and $\bar{M}_{\infty}$ have strictly less than $m$ markings of type $q$. The localization terms over 0 and $\infty$ of $\Gamma$ push forward under $\rho^{\prime \prime}$ to tautological classes by the induction hypotheses on $m$. 
If there are no refinements over $\infty$, we are in Case II of the localization formula. We then exactly follow the augmentation and exhaustion argument of the base case to conclude that

$$
\rho_{*}^{\prime \prime}\left(\omega \cap\left[\bar{M}_{\mathbf{g}, \mathbf{n}^{\prime}}\left(\bar{\beta}, \mu^{1}, \ldots, \mu^{m-1}\right)\right]^{\mathrm{vir}}\right)
$$

is tautological on the target for all relative Gromov-Witten classes $\omega$ and $\bar{\beta} \in \Pi(\mathbf{d}, \mathbf{n}, \infty)$ for all $\mathbf{n}$ subject to $d_{i} \geq\left|n_{i}\right|>0$. The induction step is therefore established and the proof of Theorem 2(ii) is complete.

2.5.5. Effectivity. The argument of Theorem 2(ii) yields an effective procedure for calculating the class of the push-forward

$$
\rho_{*}\left(\omega \cap[\bar{M}]^{\mathrm{vir}}\right)
$$

in the tautological ring of the target.

To establish the procedure, we must only check the push-forward 20 can be effectively determined if

$$
\mathbf{g}=(0, \ldots, 0)
$$

the base case of the genus induction. In fact, the argument of Theorem 2(ii) applies with almost no modification to the base case (21). A minor issue is the possibility of degenerate data over the fixed point 0 (for which the dimension formula is not valid). We leave the details to the interested reader. The outcome is a procedure for calculating 20, in the base case (21).

Theorem 2(i) is then also effective. The push-forward $\rho_{*}\left(\omega \cap\left[\bar{M}^{\dagger}\right]^{\text {vir }}\right)$ is expressible by localization in terms of the push-forwards of relative Gromov-Witten classes on unparameterized spaces.

2.5.6. Computations. Although we have established an effective procedure for calculating the classes of the push-forwards $\rho_{*}\left(\omega \cap[\bar{M}]^{\text {vir }}\right)$ and $\rho_{*}\left(\omega \cap\left[\bar{M}^{\dagger}\right]^{\text {vir }}\right)$ in the tautological ring of the target, the procedure may not be practical. The basic reason for the difficulty is the explosive growth in the number of additive generators of the tautological ring of the moduli space of curves as the number of markings increases. For example, in the case of the hyperelliptic locus, the number of additive generators of codimension $3 g$ for the tautological ring of $\bar{M}_{g, 2 g+2}$ is huge already for $g=2$.

Our purpose here is to illustrate how one can use the main result of the paper in combination with auxiliary results to obtain explicit formulas for certain classes.

By [GrP2, Prop. 11] additive generators for the tautological ring of the moduli space of curves are obtained by taking arbitrary strata, corresponding to stable graphs, and considering the push-forwards of products of arbitrary monomials in the cotangent line and $\kappa$ classes on the vertex moduli spaces. As explained in $[\mathrm{AC}]$, the pairings of such generators with monomials in the global $\kappa, \lambda$, and $\psi$ classes of the dual degree are easily written as sums of products of integrals on the vertex moduli spaces. By the Witten-Kontsevich theory, these integrals can be evaluated explicitly. For low genus, the computation of such pairings is practical. 
The null space of the pairings of the additive generators of a given degree with monomials in the $\kappa, \lambda$, and $\psi$ classes determines a space of possible relations among the generators. Often one can show (by a variety of methods) that these possible relations are actual relations, so that one obtains a basis for the tautological classes of the given degree. Then the only auxiliary information necessary to express a tautological class in terms of the basis is the evaluation of the pairings of the class with monomials in the $\kappa, \lambda$, and $\psi$ classes.

As a concrete example, consider $\overline{H E}_{g}$, the hyperelliptic locus in $\bar{M}_{g}$. By Theorem 1 , the class of $\overline{H E}_{g}$ lies in the tautological ring. In genus 3, the class of the divisor $\overline{H E}_{3}$ is well known:

$$
\left[\overline{H E}_{3}\right]_{Q}=9 \lambda_{1}-\delta_{0}-3 \delta_{1}
$$

(see $[\overline{\mathrm{HM}}]$ ). Here, we use the stack class $\left[\overline{H E}_{g}\right]_{Q}$ (see $\left.[\overline{\mathrm{M}}]\right)$. We will compute $\left[\overline{H E}_{4}\right]_{Q}$.

To begin with, by symmetry and divisor relations in low genus, one finds 14 generators for $R^{2}\left(\bar{M}_{4}\right): \kappa_{1}^{2}$ and $\kappa_{2}$ from the open stratum, the push-forwards of $\kappa_{1}$ and $\psi_{1}$ from $\bar{M}_{3,2}$ to $\Delta_{0}$, the push-forwards to $\Delta_{1}$ of $\kappa_{1}$ and $\psi_{1}$ on the genus 3 vertex, the push-forward to $\Delta_{2}$ of $\psi_{1}$ on a genus 2 vertex, and the seven strata of codimension 2 .

The classes $\kappa_{1}^{2}$ and $\kappa_{2}$ are known to be proportional in $R^{2}\left(M_{4}\right)$. Hence there is a relation in $A^{2}\left(\bar{M}_{4}\right)$ with an undetermined boundary term. The codimension 1 Chow group of a boundary divisor of $\bar{M}_{g}$ is tautological. Thus we get a relation in $R^{2}\left(\bar{M}_{4}\right)$. By intersecting with sufficiently many monomials in $\kappa$ and $\lambda$ classes, the relation is identified and shown to be unique ( $\operatorname{so} \operatorname{dim} R^{2}\left(\bar{M}_{4}\right)=13$ ). See $[\mathrm{F}]$ for an alternative derivation.

By our results in $[\mathrm{FP} \S 4]$, the pairing of $\left[\overline{H E}_{g}\right]_{Q}$ with an arbitrary monomial in $\kappa$ and $\lambda$ classes can in principle be evaluated. We have calculated the pairings with $\left[\overline{H E}_{4}\right]_{Q}$ for arbitrary monomials in $\kappa$ classes and two monomials containing a single $\lambda_{1}$. The matrix of pairings of these monomials with a basis of $R^{2}\left(\bar{M}_{4}\right)$ has rank 13 , so the class of $\overline{H E}_{4}$ can be computed.

Proposition 5. In genus 4,

$$
\begin{aligned}
2\left[\overline{H E}_{4}\right]_{Q}= & 27 \kappa_{2}-339 \lambda_{1}^{2}+64 \lambda_{1} \delta_{0}+90 \lambda_{1} \delta_{1}+6 \lambda_{1} \delta_{2}-\delta_{0}^{2} \\
& -8 \delta_{0} \delta_{1}+15 \delta_{1}^{2}+6 \delta_{1} \delta_{2}+9 \delta_{2}^{2}-4 \delta_{00}-6 \gamma_{1}+3 \delta_{01 a}-36 \delta_{11} .
\end{aligned}
$$

The strata notation of $[\bar{F}]$ is used in the above result.

\section{Nonsingularity}

\subsection{Overview}

Let $\mathbf{A}(\mathbf{d}, \mathbf{n}, k)$ be the matrix with rows and columns indexed by $\Pi(\mathbf{d}, \mathbf{n}, k)$ with the following coefficients. For $\bar{p}, \bar{q} \in \Pi(\mathbf{d}, \mathbf{n}, k)$, let the element of $\mathbf{A}(\mathbf{d}, \mathbf{n}, k)$ in position $(\bar{p}, \bar{q})$ be

$$
\prod_{i=1}^{c}\left(\prod_{j=1}^{\left|n_{i}\right|} \frac{1}{\left(p[i]_{j}\right)^{q[i]_{j}-1}} S\left[q^{\prime \prime}[i]\right]\left(p^{\prime}[i]\right)\right) \text {. }
$$

The matrix $\mathbf{A}(\mathbf{d}, \mathbf{n}, k)$ is obtained from $\mathbf{M}(\mathbf{d}, \mathbf{n}, k)$ by transposition followed by a rescaling of the rows and columns. We will prove Lemma 4 by establishing the nonsingularity of $\mathbf{A}(\mathbf{d}, \mathbf{n}, k)$. 


\subsection{Nonsingularity for $\Pi(d, n, k)$}

3.2.1. Overview. We will start by proving the nonsingularity of $\mathbf{A}$ in case the index set is $\Pi(d, n, k)$.

Lemma 5. The matrix $\mathbf{A}(d, n, k)$ is invertible.

The nonsingularity of $\mathbf{A}(\mathbf{d}, \mathbf{n}, k)$ will be a direct consequence of the proof of Lemma5.

\subsection{The function $T\left[q^{\prime \prime}\right]\left(p^{\prime \prime}\right)$}

In order to prove Lemma 5, we will require an auxiliary matrix. Let $p^{\prime \prime}$ and $q^{\prime \prime}$ be partitions with parts of size at least 2 . Define the function $T\left[q^{\prime \prime}\right]\left(p^{\prime \prime}\right)$ by the following rules:

(i) If $\ell\left(q^{\prime \prime}\right)>\ell\left(p^{\prime \prime}\right)$, then $T\left[q^{\prime \prime}\right]\left(p^{\prime \prime}\right)=0$.

(ii) If $\ell\left(q^{\prime \prime}\right) \leq \ell\left(p^{\prime \prime}\right)$, then $T\left[q^{\prime \prime}\right]\left(p^{\prime \prime}\right)$ is a sum over all injections $\theta: \underline{\ell\left(q^{\prime \prime}\right)} \rightarrow \underline{\ell\left(p^{\prime \prime}\right)}$. For such an injection $\theta$, let

$$
v(\theta)=\left|\left\{j \in \underline{\ell\left(q^{\prime \prime}\right)}: q_{j}^{\prime \prime}=p_{\theta(j)}^{\prime \prime}=2\right\}\right| .
$$

Then

$$
T\left[q^{\prime \prime}\right]\left(p^{\prime \prime}\right)=\sum_{\theta: \underline{\ell\left(q^{\prime \prime}\right) \rightarrow \underline{\ell\left(p^{\prime \prime}\right)}}} 2^{v(\theta)} \prod_{j=1}^{\ell\left(q^{\prime \prime}\right)}\left(\begin{array}{c}
p_{\theta(j)}^{\prime \prime}-1 \\
q_{j}^{\prime \prime}-1
\end{array}\right)(-1)^{q_{j}^{\prime \prime}-1}\left(q_{j}^{\prime \prime}\right)^{p_{\theta(j)}^{\prime \prime}-2} .
$$

Let $\bar{p}, \bar{q} \in \Pi(d, n, k)$. Let $p^{\prime \prime}$ and $q^{\prime \prime}$ be the subpartitions of $p^{\prime}$ and $q^{\prime}$ respectively consisting of the parts of size at least 2. Let $p^{\prime \prime \prime}$ be the subpartition of $p^{\prime \prime}$ consisting of parts at least 3 .

Let $\mathbf{B}(d, n, k)$ be the matrix with rows and columns indexed by the set $\Pi(d, n, k)$ with coefficient

$$
\frac{1}{\left|\operatorname{Aut}\left(p^{\prime \prime}\right)\right|\left|\operatorname{Aut}\left(q^{\prime \prime}\right)\right|} \prod_{h} p_{h} \prod_{i} p_{i}^{\prime \prime \prime} \prod_{j=1}^{n}\left(\left(\begin{array}{c}
p_{j}-1 \\
q_{j}-1
\end{array}\right)(-1)^{q_{j}-1} q_{j}^{p_{j}-2}\right) \cdot T\left[q^{\prime \prime}\right]\left(p^{\prime \prime}\right),
$$

in position $(\bar{p}, \bar{q})$.

\subsection{An order on $\Pi(d, n, k)$}

We order the set $\Pi(d, n, k)$ in the following manner. Let $\bar{p}$ and $\bar{q}$ be distinct elements of $\Pi(d, n, k)$. We assume the parts of the subpartitions $p^{\prime \prime}$ and $q^{\prime \prime}$ are arranged in increasing order. Then $\bar{p}$ precedes $\bar{q}$ if and only if

- $\ell\left(p^{\prime \prime}\right)<\ell\left(q^{\prime \prime}\right)$, or

- $\ell\left(p^{\prime \prime}\right)=\ell\left(q^{\prime \prime}\right)$ and $p^{\prime \prime}$ precedes $q^{\prime \prime}$ in the lexicographic order, or

- $p^{\prime \prime}=q^{\prime \prime}$ and $\ell\left(p^{\prime}\right)>\ell\left(q^{\prime}\right)$, or

- $p^{\prime}=q^{\prime}$ and $p$ precedes $q$ in the lexicographic order.

We write $\bar{p}<\bar{q}$ if $\bar{p}$ precedes $\bar{q}$ in the above order on $\Pi(d, n, k)$. 


\subsection{Proof of Lemma 5}

Define $\mathbf{C}(d, n, k)$ by

$$
\mathbf{C}(d, n, k)=\mathbf{B}(d, n, k) \cdot \mathbf{A}(d, n, k) .
$$

Lemma 5 is an immediate consequence of the following result.

Lemma 6. The matrix $\mathbf{C}(d, n, k)$ is upper triangular with 1 's along the diagonal.

Proof. Recall the well known identities

$$
\sum_{k=0}^{n}\left(\begin{array}{l}
n \\
k
\end{array}\right)(-1)^{k} k^{a}=0, \quad 0 \leq a \leq n-1,
$$

and

To prove these identities, consider the function

$$
\sum_{k=0}^{n}\left(\begin{array}{l}
n \\
k
\end{array}\right)(-1)^{k}(k+1)^{-1}=\frac{1}{n+1}, \quad n \geq 0 .
$$

$$
f(t)=\sum_{k=0}^{n}\left(\begin{array}{l}
n \\
k
\end{array}\right)(-1)^{k} t^{k}=(1-t)^{n} .
$$

The first identity is obtained by the evaluation of $\left(t \frac{d}{d t}\right)^{a} f$ at $t=1$, and the second is obtained by computing $\int_{0}^{1} f(t) d t$.

Let $\tilde{p}, \tilde{r}, \hat{p}$, and $\hat{r}$ be integers satisfying $\tilde{p} \geq \tilde{r} \geq 1$ and $\hat{p} \geq \hat{r} \geq 2$. Further, let $\delta(x, y, z)=1$ when $x=y=z$, and 0 otherwise. We will need four closely related sums:

$$
\begin{aligned}
& (\alpha): \quad \sum_{\tilde{q}=1}^{\tilde{p}}\left(\begin{array}{l}
\tilde{p}-1 \\
\tilde{q}-1
\end{array}\right)(-1)^{\tilde{q}-1} \tilde{q}^{\tilde{p}-1-\tilde{r}}= \begin{cases}0, & \tilde{r}<\tilde{p} \\
1 / \tilde{p}, & \tilde{r}=\tilde{p}\end{cases} \\
& (\beta): \quad \sum_{\hat{q}=1}^{\hat{p}}\left(\begin{array}{l}
\hat{p}-1 \\
\hat{q}-1
\end{array}\right)(-1)^{\hat{q}-1} \hat{q}^{\hat{p}-3} 2^{\delta(2, \hat{p}, \hat{q})}= \begin{cases}0, & \hat{p} \geq 3 \\
0, & \hat{p}=2\end{cases} \\
& \left(\beta^{\prime}\right): \quad \sum_{\hat{q}=1}^{\hat{p}}\left(\begin{array}{l}
\hat{p}-1 \\
\hat{q}-1
\end{array}\right)(-1)^{\hat{q}-1} \hat{q}^{\hat{p}-2} 2^{\delta(2, \hat{p}, \hat{q})}= \begin{cases}0, & \hat{p} \geq 3, \\
-1, & \hat{p}=2\end{cases} \\
& (\gamma): \quad \sum_{\hat{q}=1}^{\hat{p}}\left(\begin{array}{l}
\hat{p}-1 \\
\hat{q}-1
\end{array}\right)(-1)^{\hat{q}-1} \hat{q}^{\hat{p}-1-\hat{r}} 2^{\delta(2, \hat{p}, \hat{q})}= \begin{cases}0, & \hat{r}<\hat{p}, \\
0, & \hat{r}=\hat{p}=2, \\
1 / \hat{p}, & \hat{r}=\hat{p} \geq 3 .\end{cases}
\end{aligned}
$$

Let $\bar{p}$ and $\bar{r}$ be elements of $\Pi(d, n, k)$. The matrix element $\mathbf{C}(\bar{p}, \bar{r})$ is given by

$$
\begin{aligned}
& \mathbf{C}(\bar{p}, \bar{r})=\sum_{\bar{q} \in \Pi(d, n, k)} \mathbf{B}(\bar{p}, \bar{q}) \mathbf{A}(\bar{q}, \bar{r}) \\
& =\frac{\prod p_{h} \prod p_{i}^{\prime \prime \prime}}{\left|\operatorname{Aut}\left(p^{\prime \prime}\right)\right|} \sum_{\bar{q}} \prod_{j=1}^{n}\left(\left(\begin{array}{l}
p_{j}-1 \\
q_{j}-1
\end{array}\right)(-1)^{q_{j}-1} q_{j}^{p_{j}-1-r_{j}}\right) \cdot \frac{T\left[q^{\prime \prime}\right]\left(p^{\prime \prime}\right)}{\left|\operatorname{Aut}\left(q^{\prime \prime}\right)\right|} \cdot S\left[r^{\prime \prime}\right]\left(q^{\prime}\right) .
\end{aligned}
$$

We must show: 
(i) $\mathbf{C}(\bar{p}, \bar{r})=0$ if $\bar{r}$ precedes $\bar{p}$ in the order on $\Pi(d, n, k)$,

(ii) $\mathbf{C}(\bar{p}, \bar{p})=1$ for all $\bar{p}$.

Let $\bar{r} \leq \bar{p}$ with respect to the order on $\Pi(d, n, k)$. The prefactor $\prod p_{h} \prod p_{i}^{\prime \prime \prime} /\left|\operatorname{Aut}\left(p^{\prime \prime}\right)\right|$ plays a role only in the normalization of the diagonal elements (ii). We will first concentrate on the main sum in formula 22 for $\mathbf{C}(\bar{p}, \bar{r})$ : a triple sum over partially ordered partitions $\bar{q}$, injections $\theta: \underline{\ell\left(q^{\prime \prime}\right)} \rightarrow \underline{\ell\left(p^{\prime \prime}\right)}$ via $T\left[q^{\prime \prime}\right]\left(p^{\prime \prime}\right)$, and injections $\iota: \underline{\ell\left(r^{\prime \prime}\right)} \rightarrow \underline{\ell\left(q^{\prime}\right)}$ via $S\left[r^{\prime \prime}\right]\left(q^{\prime}\right)$.

The key step in the proof is to recognize that the main sum in 22 can be written in terms of products of the sums $(\alpha),(\beta),\left(\beta^{\prime}\right)$, and $(\gamma)$ discussed above. The products have $n+\left|p^{\prime \prime}\right|$ factors. We will define and analyze the products in stages.

We first consider the case $p^{\prime \prime}=\emptyset$. We will write the sum 22 as a single product with $n$ factors of type $(\alpha)$. The $n$ factors are obtained by replacing $(\tilde{p}, \tilde{q}, \tilde{r})$ in the formula for $(\alpha)$ by $\left(p_{i}, q_{i}, r_{i}\right)$ for $1 \leq i \leq n$. As $q_{i}$ varies in the range $1 \leq q_{i} \leq p_{i}$, all possible choices of the ordered partition $q=\left(q_{1}, \ldots, q_{n}\right)$, in the partially ordered partition $\bar{q}=$ $\left(q, q^{\prime}\right)$ indexing 22, occur, as $\bar{q}$ does not contribute to 22 if there exists an $i$ for which $q_{i}>p_{i}$.

Since $p^{\prime \prime}=\emptyset$, we find that for $\bar{q}$ to contribute to 22, the equality $q^{\prime \prime}=\emptyset$ must hold. By the ordering $\bar{r} \leq \bar{p}$, the equality $r^{\prime \prime}=\emptyset$ must hold. The sum over $\bar{q}, \theta$, and $\iota$ is then just a sum over $\bar{q}$. The partially ordered partition $\bar{q}$ is given by the choice of $q=\left(q_{1}, \ldots, q_{n}\right)$, for $q^{\prime}$ then consists of $d-\sum q_{i}$ parts equal to 1 . We conclude that the main sum of 22 equals the product of the $n$ sums of type $(\alpha)$.

The product of the $n$ sums vanishes if there exists an $i$ for which $r_{i}<p_{i}$. Since $\bar{r} \leq \bar{p}$ and $r^{\prime \prime}=p^{\prime \prime}$, we find $\left|r^{\prime}\right| \geq\left|p^{\prime}\right|$. Hence, $\sum_{i=1}^{n} r_{i} \leq \sum_{i=1}^{n} p_{i}$. For a nonvanishing product, $\bar{r}=\bar{p}$ is then necessary. In the diagonal case, the product equals $\prod_{i=1}^{n}\left(1 / p_{i}\right)$, which is exactly cancelled by the prefactor to yield the matrix element 1 . We have completed the analysis of the case $p^{\prime \prime}=\emptyset$.

We now consider general $p^{\prime \prime}$. Let $m=\ell\left(p^{\prime \prime}\right)$. We will analyze associated products with $n$ factors of type $(\alpha)$ and $m$ additional factors, each of type $(\beta),\left(\beta^{\prime}\right)$, or $\gamma$. The first $n$ factors are obtained as before by replacing $(\tilde{p}, \tilde{q}, \tilde{r})$ in the formula for $(\alpha)$ by $\left(p_{i}, q_{i}, r_{i}\right)$, for $1 \leq i \leq n$. Let the $m$ upper bounds of the last sums be denoted by $\hat{p}_{1}, \ldots, \hat{p}_{m}$, and let the $m$ index variables for the sums be $\hat{q}_{1}, \ldots, \hat{q}_{m}$. The precise forms of the last $m$ sums will be specified below.

Let $\hat{p}_{i}=p_{i}^{\prime \prime}$ for $1 \leq i \leq m$. The choice of the $m$ index variables $\hat{q}_{1}, \ldots, \hat{q}_{m}$ exactly corresponds to the choice of a $\bar{q}$ contributing to 22) together with an injection $\theta$, up to automorphisms of $q^{\prime \prime}$. More precisely, as the index variables of the first $n$ sums determine $q=\left(q_{1}, \ldots, q_{n}\right)$, the unordered partition $q^{\prime}$ consists of $\hat{q}_{1}, \ldots, \hat{q}_{m}$ and

$$
d-\sum_{i=1}^{n} q_{i}-\sum_{j=1}^{m} \hat{q}_{j}
$$

parts equal to 1 . Clearly, $q^{\prime \prime}$ consists then of those $\hat{q}_{j}$ that are at least 2 . Finally, the $j$ such that $\hat{q}_{j} \geq 2$ determine $\operatorname{Im}(\theta)$, and $\theta$ itself is uniquely determined up to automorphisms of $q^{\prime \prime}$. 
Let us analyze the factor $S\left[r^{\prime \prime}\right]\left(q^{\prime}\right)$. Let $q^{*}$ denote the subpartition of $q^{\prime}$ consisting of $\hat{q}_{1}, \ldots, \hat{q}_{m}$. Fix an injection $\iota: \underline{\ell\left(r^{\prime \prime}\right)} \rightarrow \underline{\ell\left(q^{\prime}\right)}$. Suppose $c$ of the $m$ parts of $q^{*} \operatorname{lie}$ in $\operatorname{Im}(\iota)$. The summand

$$
\prod_{j=1}^{\ell\left(r^{\prime \prime}\right)} \frac{1}{\left(q_{l(j)}^{\prime}\right)^{r_{j}^{\prime \prime}-1}} \cdot \prod_{i \notin \operatorname{Im}(\iota)} \frac{1}{q_{i}^{\prime}}
$$

of $S\left[r^{\prime \prime}\right]\left(q^{\prime}\right)$ corresponding to $\iota$ can, in first approximation, be accounted for by taking $m-c$ factors of type $(\beta)$ and $c$ factors of type $(\gamma)$. The last $c$ factors correspond exactly to the $c$ parts of $q^{*}$ in $\operatorname{Im}(\iota)$, and the constants $\hat{r}$ in the factors are set to equal the parts $r_{j}^{\prime \prime}$.

Given $\iota$, we have defined an initial product of $n+m$ factors of type $(\alpha),(\beta)$, and $(\gamma)$. However, the terms of the product do not properly account for all the data of $\iota$. The difficulty is that while $\iota$ specifies the subinjection of the $\ell\left(r^{\prime \prime}\right)-c$ parts of $r^{\prime \prime}$ to the parts of $q^{\prime}$ not in $q^{*}$, the terms of the defined product do not.

We must alter the product in order to properly account for $\iota$. The partition $q^{\prime}$ consists of $q^{*}$ and

$$
e=d-\sum_{i=1}^{n} q_{i}-\sum_{j=1}^{m} \hat{q}_{j}
$$

parts equal to 1 . The number of subinjections of the remaining $\ell\left(r^{\prime \prime}\right)-c$ parts of $r^{\prime \prime}$ into the $e$ parts of $q^{\prime}$ not in $q^{*}$ equals

$$
e ! /\left(e-\ell\left(r^{\prime \prime}\right)+c\right) !
$$

By giving such a subinjection in addition to the other data, the injection $\iota$ is essentially determined. To be precise, by the choice of $c$ factors of type $(\gamma)$, the choice of $c$ corresponding parts $r_{j}^{\prime \prime}$, and the choice of a subinjection of the remaining $\ell\left(r^{\prime \prime}\right)-c$ parts of $r^{\prime \prime}$ into the $e$ parts of $q^{\prime}$ not in $q^{*}$, the injection $\iota$ is uniquely determined up to automorphisms of the $c$ chosen parts $r_{j}^{\prime \prime}$.

Consider the $e ! /\left(e-\ell\left(r^{\prime \prime}\right)+c\right)$ ! subinjections into the $e$ parts of $q^{\prime}$ not in $q^{*}$, with

$$
e=d-\sum_{i=1}^{n} q_{i}-\sum_{j=1}^{m} \hat{q}_{j}
$$

A crucial observation is the following: we can take account of the subinjections by slight modifications of the factors of type $(\alpha),(\beta)$, and $(\gamma)$. Namely, after expanding $e ! /(e-$ $\left.\ell\left(r^{\prime \prime}\right)+c\right)$ ! we find a polynomial $f$ of degree $\ell\left(r^{\prime \prime}\right)-c$ in $q_{i}$ and $\hat{q}_{j}$. Each $q_{i}$ raises the exponent of $q_{i}$ in the $i$ th factor of type $(\alpha)$ by 1 . Similarly, each $\hat{q}_{j}$ raises the exponent of $\hat{q}_{j}$ in the $j$ th factor of type either $(\beta)$ or $(\gamma)$ by 1 . After raising, a factor of type $(\beta)$ becomes a factor of type $\left(\beta^{\prime}\right)$.

Starting with an injection $\iota$, we have obtained, by expanding $f$, a sum of products. Each product consists of factors (possibly raised) of types $(\alpha),(\beta)$, and $(\gamma)$. The sum of products defines a subsum of

$$
\sum_{\bar{q}} \prod_{j=1}^{n}\left(\left(\begin{array}{c}
p_{j}-1 \\
q_{j}-1
\end{array}\right)(-1)^{q_{j}-1} q_{j}^{p_{j}-1-r_{j}}\right) \cdot \frac{T\left[q^{\prime \prime}\right]\left(p^{\prime \prime}\right)}{\left|\operatorname{Aut}\left(q^{\prime \prime}\right)\right|} \cdot S\left[r^{\prime \prime}\right]\left(q^{\prime}\right) .
$$


For example, the factor $2^{v(\theta)}$ in $T\left[q^{\prime \prime}\right]\left(p^{\prime \prime}\right)$ has been rewritten as a product of the factors $2^{\delta\left(2, \hat{p}_{j}, \hat{q}_{j}\right)}$ occurring in the (possibly raised) sums $(\beta)$ and $(\gamma)$.

We may now finally establish the required vanishing and nonvanishing. Since $\bar{r} \leq \bar{p}$, we have

$$
\ell\left(r^{\prime \prime}\right) \leq \ell\left(p^{\prime \prime}\right)=m .
$$

The initial product obtained from $\iota$ had $n$ factors of type $(\alpha), m-c$ factors of type $(\beta)$, and $c$ factors of type $(\gamma)$. Every factor of type $(\beta)$ vanishes. Every such factor must be raised for a nonvanishing contribution. The maximum number of raisings is $\ell\left(r^{\prime \prime}\right)-c$. So for nonvanishing, $\ell\left(r^{\prime \prime}\right)=m$ is required. Moreover, every factor of type $(\beta)$ becomes a factor of type $\left(\beta^{\prime}\right)$ with a minus sign. The product of $n$ factors of type $(\alpha), m-c$ factors of type $\left(\beta^{\prime}\right)$, and $c$ factors of type $(\gamma)$, the unique possibly nonvanishing contribution, appears with a coefficient $(-1)^{m-c}(m-c)$ !.

Since $\ell\left(r^{\prime \prime}\right)=\ell\left(p^{\prime \prime}\right)$, the partition $r^{\prime \prime}$ must precede $p^{\prime \prime}$ in the lexicographic order. So for every part of $p^{\prime \prime}$ equal to 2, there exists a part of $r^{\prime \prime}$ equal to 2. To obtain a nonvanishing contribution, each such part of $p^{\prime \prime}$ must come with a factor of type $\left(\beta^{\prime}\right)$. Considering the parts of $p^{\prime \prime}$ equal to $3,4,5, \ldots$ successively, we find a nonvanishing contribution requires that each such part come with a factor of type $(\gamma)$ and an equal part of $r^{\prime \prime}$. Thus, $r^{\prime \prime}=p^{\prime \prime}$. Arguing as in the case $r^{\prime \prime}=p^{\prime \prime}=\emptyset$, we find that a nonvanishing contribution requires $\bar{r}=\bar{p}$. We have proven the required vanishing when $\bar{r}<\bar{p}$.

For the diagonal elements, assume $\bar{r}=\bar{p}$. The only nonvanishing contributions arise by exactly matching every part of $p^{\prime \prime \prime}$ with an equal part of $r^{\prime \prime}$ (via the element of $q^{\prime}$ in the corresponding factor of type $(\gamma)$ ), inserting a factor of type $\left(\beta^{\prime}\right)$ for each remaining 2 in $p^{\prime \prime}$, and mapping the 2's in $r^{\prime \prime}$ to the 1's of $q^{\prime}$ not in $q^{*}$. The number of automorphisms of the $c$ chosen parts $r_{j}^{\prime \prime}$ may be identified with $\left|\operatorname{Aut}\left(p^{\prime \prime \prime}\right)\right|$. The factor $(m-c)$ ! may now be identified with the number of permutations of the 2's. Multiplied together, these factors yield $\left|\operatorname{Aut}\left(p^{\prime \prime}\right)\right|$, cancelling the denominator of the prefactor of the matrix element $\mathbf{C}(\bar{p}, \bar{p})$. Finally, the factor $(-1)^{m-c}$ cancels the contribution of the $m-c$ factors of type $\left(\beta^{\prime}\right)$, and the numerator $\prod p_{h} \prod p_{i}^{\prime \prime \prime}$ of the leading factor cancels the contribution of the factors of type $(\alpha)$ and $(\gamma)$. We conclude $C(\bar{p}, \bar{p})=1$ for all $\bar{p}$.

The lower bound $d-k$ for the length of partitions $\bar{p}$ in $\Pi(d, n, k)$ does not play a crucial role. In fact, Lemmas 5 and 6 hold for the submatrices of $\mathbf{A}(d, n, \infty)$ and $\mathbf{B}(d, n, \infty)$ indexed by any subset $\Xi$ of $\Pi(d, n, \infty)$ for which

$$
\Pi_{\bar{p}}=\left\{\bar{q} \in \Pi(d, n, \infty): q \leq p, 1^{\ell\left(p^{\prime \prime}\right)-\ell\left(q^{\prime \prime}\right)} q^{\prime \prime} \leq p^{\prime \prime}\right\}
$$

is contained in $\Xi$ for all $\bar{p} \in \Xi$. The proof is obtained simply by analyzing the vanishing of entries of $\mathbf{B}(d, n, \infty)$ forced by the definition of the matrix (and the definition of $T$ ).

For Lemmas 5 and 6 , the set $\Xi$ should be closed under the operation of lowering the parts of a partially ordered partition, necessarily making up for this by adding parts equal to 1 to the unordered partition. Clearly, the subsets $\Pi(d, n, k)$ satisfy this condition for all $k \geq 0$. 


\subsection{The nonsingularity of $\mathbf{A}(\mathbf{d}, \mathbf{n}, k)$}

The nonsingularity of $\mathbf{A}(\mathbf{d}, \mathbf{n}, k)$ is immediate when

$$
k \geq d-\sum_{i=1}^{c}\left|n_{i}\right|,
$$

for then the matrix is the Kronecker product of the matrices $A\left(d_{i},\left|n_{i}\right|, d_{i}-\left|n_{i}\right|\right)$. For smaller $k$, certain rows and the corresponding columns are omitted and the resulting matrix is not necessarily a Kronecker product. However, for each $k$ the set $\Pi(\mathbf{d}, \mathbf{n}, k)$ is closed under the operation of lowering the parts of some of the constituent partially ordered partitions and compensating by adding parts equal to 1 to the corresponding unordered partitions. From the remark at the end of 3.5 , the method of proof of Lemmas 5 and 6 still applies.

The nonsingularity of $\mathbf{A}(\mathbf{d}, \mathbf{n}, k)$ is established and the proof of Lemma 4 is complete.

\section{Consequences}

\subsection{Proof of Proposition 3}

4.1.1. The moduli space $\bar{M}_{g, n}$. We prove that the socle $R^{3 g-3+n}\left(\bar{M}_{g, n}\right)$ is 1-dimensional. By [GrP2, Proposition 11], the socle is generated by classes of the form

$$
\xi_{B *}\left(\prod_{v \in V(B)} \theta_{v}\right),
$$

where $B$ is a stable graph of genus $g$ with $n$ legs and $\theta_{v} \in R^{*}\left(\bar{M}_{g(v), n(v)}\right)$ is a monomial in the $\psi$ and $\kappa$ classes of $\bar{M}_{g(v), n(v)}$, of degree equal to the dimension $3 g(v)-3+n(v)$. By Proposition 2, we may assume

$$
3 g(v)-3+n(v) \leq g(v)-1+\delta_{0 g}(v)
$$

for all $v \in V(B)$. This implies $g(v)=0$ and $n(v)=3$. So $R^{3 g-3+n}\left(\bar{M}_{g, n}\right)$ is generated by the point classes of maximally degenerate stable $n$-pointed curves of genus $g$. All such curves are degenerations of stable $n$-pointed irreducible rational curves (with $g$ nodes). The closed stratum of such curves is dominated by $\bar{M}_{0,2 g+n}$. Hence all the point classes are equivalent, which proves the socle claim for $\bar{M}_{g, n}$.

4.1.2. The moduli space $M_{g, n}^{\mathrm{c}}$. The maximally degenerate stable $n$-pointed curves of genus $g$ of compact type consist of $g$ elliptic tails attached to $g$ of the marked points of a maximally degenerate stable $(g+n)$-pointed curve of genus 0 (contracted to a point when $g+n=2$ ). The classes of the different strata determined by these maximally degenerate curves are rationally equivalent in $M_{g, n}^{\mathrm{c}}$ via the rational equivalence of points on $\bar{M}_{0, g+n}$. We prove that these strata classes generate the socle and that classes of higher degree vanish. 
The tautological ring $R^{*}\left(M_{g, n}^{\mathrm{c}}\right)$ is additively generated by classes of the form $\xi_{B *}\left(\prod_{v \in V(B)} \theta_{v}\right)$, where $B$ is now a stable tree of genus $g$ with $n$ legs. By Proposition 2 . we may assume

$$
\operatorname{deg} \theta_{v} \leq g(v)-1+\delta_{0 g(v)}-\delta_{0 n(v)}
$$

Since

$$
g(v)-1+\delta_{0 g(v)}-\delta_{0 n(v)} \leq 2 g(v)-3+n(v),
$$

we find

$$
\begin{aligned}
\operatorname{deg} \xi_{B *}\left(\prod_{v \in V(B)} \theta_{v}\right) & =-1+\sum_{v \in V(B)}\left(1+\operatorname{deg} \theta_{v}\right) \\
& \leq-1+\sum_{v \in V(B)}\left(g(v)+\delta_{0 g(v)}-\delta_{0 n(v)}\right) \\
& \leq-1+\sum_{v \in V(B)}(2 g(v)-2+n(v))=2 g-3+n,
\end{aligned}
$$

which proves the vanishing. The equality

$$
\operatorname{deg} \xi_{B *}\left(\prod_{v \in V(B)} \theta_{v}\right)=2 g-3+n
$$

implies that $(g(v), n(v))=(0,3)$ or $(1,1)$ for all $v$, proving the socle claim for $M_{g, n}^{\mathrm{c}}$.

4.1.3. The moduli space $M_{g, n}^{\mathrm{rt}}$ for $g \geq 2$. The tautological ring is additively generated by classes of the form $\xi_{B *}\left(\prod_{v \in V(B)} \theta_{v}\right)$, where $B$ is now a stable tree of genus $g$ with $n$ legs and a single vertex $w$ of genus $g$ (and all other vertices of genus 0 ). By Proposition 2 , we may assume the class $\theta_{v}$ has degree at most $g-1-\delta_{0 n}$ on $w$ (and degree 0 on the genus 0 vertices). Then

$$
\begin{aligned}
\operatorname{deg} \xi_{B *}\left(\prod_{v \in V(B)} \theta_{v}\right) & =-1+\sum_{v \in V(B)}\left(1+\operatorname{deg} \theta_{v}\right) \leq g-1-\delta_{0 n}-1+\sum_{v \in V(B)} 1 \\
& \leq g-1-\delta_{0 n}+\sum_{v \neq w}(n(v)-2)=g-1-\delta_{0 n}+n-n(w) \\
& \leq g-2+n,
\end{aligned}
$$

which proves the vanishing. The equality

$$
\operatorname{deg} \xi_{B *}\left(\prod_{v \in V(B)} \theta_{v}\right)=g-2+n
$$

implies $n(v)=3$ for $v \neq w$, that $n(w)=0$ or 1 , and $\theta_{w}$ is a top class on $M_{g}$ or $M_{g, 1}$. The 1-dimensionality of the socle follows now from Looijenga's results [Lo] for $M_{g}$ and $M_{g, 1}$, the nonvanishing of $\kappa_{g-2}$ (see $[\overline{\mathrm{FP}}]$ ), and the rational equivalence of points on $\bar{M}_{0, n+1}$. 


\subsection{Gromov-Witten theory}

4.2.1. $\kappa$ descendent invariants. Let $X$ be a nonsingular projective variety. The $\kappa$ descendent Gromov-Witten invariants of $X$ are defined by:

$$
\left\langle\tau_{e_{1}}\left(\gamma_{1}\right) \cdots \tau_{e_{n}}\left(\gamma_{n}\right) \prod_{j \geq 0} \kappa_{j}^{f_{j}}\right\rangle_{g, n, \beta}^{X}=\int_{\left[\bar{M}_{g, n}(X, \beta)\right]^{\mathrm{vir}}} \prod_{i=1}^{n} \psi_{i}^{e_{i}} \operatorname{ev}_{i}^{*}\left(\gamma_{i}\right) \prod_{j \geq 0} \kappa_{j}^{f_{j}},
$$

where $\psi_{i}$ is the cotangent line on the domain, $\operatorname{ev}_{i}^{*}\left(\gamma_{i}\right)$ is the pull-back of $\gamma_{i} \in H^{*}(X, \mathbb{Q})$ via the $i$ th evaluation map, and $\kappa_{j}$ is the Arbarello-Cornalba $\kappa$ class on the moduli space of maps.

4.2.2. Proof of Proposition 4 Since Proposition 4 is well known in genus 0 and 1, we will assume $g \geq 2$. Let

$$
\rho: \bar{M}_{g, n}(X, \beta) \rightarrow \bar{M}_{g, n} .
$$

By the comparison results relating $\psi_{i}$ and $\kappa_{j}$ to the $\rho$-pull-backs of the corresponding classes on the moduli space of curves, the invariants 23 are equal to the integrals

$$
\int_{\left[\bar{M}_{g, n}(X, \beta)\right]^{\mathrm{vir}}} \prod_{i=1}^{n} \rho^{*}\left(\psi_{i}^{e_{i}}\right) \mathrm{ev}_{i}^{*}\left(\gamma_{i}\right) \prod_{j \geq 0} \rho^{*}\left(\kappa_{j}^{f_{j}}\right),
$$

modulo corrections by invariants indexed by lower data $(g, n, \beta)$. Here, $\psi_{i}$ and $\kappa_{j}$ denote the cotangent line and Arbarello-Cornalba $\kappa$ class on the moduli space of curves. We will prove Proposition 4 for the integrals (24). The result for the invariants (23) follows.

Let $\sum_{i=1}^{n} e_{i}+\sum_{j \geq 0} j f_{j} \geq g$. Then, by Proposition 2 the class

$$
\prod_{i=1}^{n} \psi_{i}^{e_{i}} \prod_{j \geq 0} \kappa_{j}^{f_{j}}
$$

on $\bar{M}_{g, n}$ can be rewritten as a tautological boundary class. The boundary strata are indexed by dual graphs. Basic tautological classes are obtained on a given stratum by products of $\psi$ and $\kappa$ classes,

$$
\prod_{i=1}^{\operatorname{val}(v)} \psi_{i}^{a_{i}} \prod_{j \geq 0} \kappa_{j}^{b_{j}},
$$

at each vertex $v$ of the dual graph. Every tautological boundary class is a linear combination of these basic classes (see [GrP2]). By Proposition 2, we may require the condition

$$
\sum_{i=1}^{\operatorname{val}(v)} a_{i}+\sum_{j \geq 0} j b_{j}<g(v)+\delta_{0 g(v)}
$$

at each vertex $v$.

The proof of Proposition 4 is completed by rewriting (25) as a sum of basic classes satisfying the vertex condition [26) and then applying the splitting axiom to 24]. 


\subsection{The generation conjecture}

We conjecture the ring $R^{*}\left(M_{g, n}\right)$ is additively generated by a restricted set of tautological classes.

Conjecture 4. $R^{*}\left(M_{g, n}\right)$ is additively generated by monomials

$$
\prod_{i=1}^{n} \psi_{i}^{e_{i}} \prod_{j \geq 1} \kappa_{j}^{f_{j}}
$$

satisfying

$$
\sum_{i=1}^{n} e_{i}+\sum_{j \geq 1}(j+1) f_{j}<g+\delta_{0 g} .
$$

We have verified Conjecture 4 for $g+3 n \leq 21$, assuming for $n>0$ the Gorenstein conjecture for $\mathcal{C}_{g}^{n}$, the fiber product of the universal curve over $M_{g}$.

Conjecture 3 is a consequence of the following three statements: Proposition 4 , Conjecture 4 , and the (unproven) exactness of the sequence

$$
R^{*}\left(\partial \bar{M}_{g, n}\right) \rightarrow R^{*}\left(\bar{M}_{g, n}\right) \rightarrow R^{*}\left(M_{g, n}\right) \rightarrow 0 .
$$

Conjecture 3 is derived from these statements by the standard method of expressing $\kappa$ monomials in terms of push-forwards of cotangent line classes on additionally pointed spaces. On $\bar{M}_{g, n}$, the class

$$
\prod_{j \geq 1} \kappa_{j}^{f_{j}}
$$

is expressed as a sum of classes

$$
\pi_{r *}\left(\prod_{i=1}^{r} \psi_{n+i}^{h_{i}}\right)
$$

where $\pi_{r}: \bar{M}_{g, n+r} \rightarrow \bar{M}_{g, n}$ is the forgetting map and

$$
r \leq \sum_{j \geq 1} f_{j}, \quad \sum_{i=1}^{r} h_{i} \leq \sum_{j \geq 1}(j+1) f_{j}
$$

(see $[\overline{\mathrm{AC}}]$ ). We leave the details to the reader.

Acknowledgements. We thank T. Graber, A. Okounkov, Y. Ruan, and R. Vakil for discussions of the relative Gromov-Witten theory of $\mathbf{P}^{1}$. We thank the referee for several useful suggestions. The research reported here was pursued during a visit by C.F. to Princeton University in the spring of 2003. R.P. was partially supported by DMS-0236984 and fellowships from the Sloan and Packard foundations.

\section{References}

[AC] Arbarello, E., Cornalba, M.: Combinatorial and algebro-geometric cohomology classes on the moduli spaces of curves. J. Algebraic Geom. 5, 705-749(1996) Zbl 0886.14007 MR 99c:14033 
[BP] Belorousski, P., Pandharipande, R.: A descendent relation in genus 2. Ann. Scuola Norm. Sup. Pisa Cl. Sci. (4) 29, 171-191 (2000) Zbl 0981.81063 MR 2001g:14044

[F] Faber, C.: Chow rings of moduli spaces of curves. II. Some results on the Chow ring of $\bar{M}_{4}$. Ann. of Math. (2) 132, 421-449 (1990) Zbl 0735.14021 MR 91h:14009b

[FP] Faber, C., Pandharipande, R.: Logarithmic series and Hodge integrals in the tautological ring. With an appendix by Don Zagier. Michigan Math. J. 48, 215-252 (2000) Zbl pre01700870 MR 2002e:14041

[Ge1] Getzler, E.: Intersection theory on $\bar{M}_{1,4}$ and elliptic Gromov-Witten invariants. J. Amer. Math. Soc. 10, 973-998 (1997) Zbl 0909.14002 MR 98f:14018

[Ge2] Getzler, E.: Topological recursion relations in genus 2. In: Integrable Systems and Algebraic Geometry (Kobe/Kyoto, 1997), World Sci., River Edge, NJ, 73-106 (1998) Zbl 1021.81056 MR 2000b:14028

[GrP1] Graber, T., Pandharipande, R.: Localization of virtual classes. Invent. Math. 135, 487518 (1999) Zbl 0953.14035 MR 2000h:14005

[GrP2] Graber, T., Pandharipande, R.: Constructions of nontautological classes on moduli spaces of curves. Michigan Math. J. 51, 93-109 (2003) Zbl pre01943247 MR 2004e:14043

[GrV1] Graber, T., Vakil, R.: On the tautological ring of $\bar{M}_{g, n}$. Turkish J. Math. 25, 237-243 (2001) Zbl pre01610289 MR 2002b:14034

[GrV2] Graber, T., Vakil, R.: Relative virtual localization and vanishing of tautological classes on moduli spaces of curves. math.AG/0309227.

[HM] Harris, J., Mumford, D.: On the Kodaira dimension of the moduli space of curves. With an appendix by William Fulton. Invent. Math. 67, 23-88 (1982) Zbl 0506.14016 MR 83i:14018

[I] Ionel, E.-N.: Topological recursive relations in $H^{2 g}\left(M_{g, n}\right)$. Invent. Math. 148, 627-658 (2002) Zbl pre01777257 MR 2003d:14065

[K] Keel, S.: Intersection theory of moduli space of stable $n$-pointed curves of genus zero. Trans. Amer. Math. Soc. 330, 545-574 (1992) Zbl 0768.14002 MR 92f:14003

[Li] Li, J.: Stable morphisms to singular schemes and relative stable morphisms. J. Differential Geom. 57, 509-578 (2001) Zbl pre01782688 MR 2003d:14066

[LR] Li, A.-M., Ruan, Y.: Symplectic surgery and Gromov-Witten invariants of Calabi-Yau 3-folds. Invent. Math. 145, 151-218 (2001) Zbl pre01655744 MR 2002g:53158

[Lo] Looijenga, E.: On the tautological ring of $M_{g}$. Invent. Math. 121, 411-419 (1995) Zbl 0851.14017 MR 96g:14021

[M] Mumford, D.: Towards an enumerative geometry of the moduli space of curves. In: Arithmetic and Geometry, Vol. II, Progr. Math. 36, Birkhäuser Boston, Boston, MA, 271-328 (1983) Zbl 0554.14008 MR 85j:14046

[P1] Pandharipande, R.: A geometric construction of Getzler's elliptic relation. Math. Ann. 313, 715-729 (1999) Zbl 0933.14035 MR 2000d:14059

[P2] Pandharipande, R.: Three questions in Gromov-Witten theory. In: Proc. of the ICM 2002 Beijing, Vol. II, 503-512 Zbl pre01789991 MR 2003k:14069 